\title{
The Growth Hormone Releasing Hormone Signaling Pathway Governs Cardiomyocyte differentiation in human iPS cells
}

Amarylis C.B.A. Wanschel ${ }^{1 \S}$, Konstantinos E. Hatzistergos ${ }^{\star} § 1,5,7$, Alessandro G. Salerno $^{1}$, Jeffim

N. Kuznetsov ${ }^{1,2}$, Stefan Kurtenbach ${ }^{1,2}$, Daniel A. Rodriguez ${ }^{1,2}$, Krystalenia Valasaki ${ }^{1}$, Wayne Balkan $^{1,3}$, Derek Dykxhoorn ${ }^{1,4}$, Andrew V. Schally $y^{1,3,6}$, \& Joshua M. Hare ${ }^{1,3^{*}}$

${ }^{1}$ Interdisciplinary Stem Cell Institute and Departments of ${ }^{2}$ Ophthamology, ${ }^{3}$ Medicine, ${ }^{4}$ Human Genetics and ${ }^{5}$ Cell Biology, University of Miami Miller School of Medicine, Miami, FL, USA.

${ }^{6}$ Endocrine, Polypeptide and Cancer Institute, Veteran Affairs Medical Center, Miami, FL

7 Department of Genetics, Development and Molecular Biology, Aristotle University of Thessaloniki, Thessaloniki, Greece.

§Equal contribution.

${ }^{*}$ Corresponding Authors:

Joshua M. Hare, MD

University of Miami Miller School of Medicine

Interdisciplinary Stem Cell Institute, Biomedical Research Building, Room 908

1501 N.W. 10th Ave, P.O Box 016960 (R125)

Miami, FI, 33136

Tel: 305-243-1999

email: $\underline{\text { JHare@med.miami.edu }}$

Konstantinos Hatzistergos, PhD

Faculty of Sciences - School of Biology - Aristotle University of Thessaloniki

Department of Genetics, Development and Molecular Biology

Thessaloniki, Greece, 54124

Tel: +30 2310998257

Email: kchatzistergos@bio.auth.gr 
Conflict of Interest Statement: Dr. Andrew V. Schally and Dr. Joshua M. Hare are listed as coinventors on patents on GHRH analogs which were assigned to the University of Miami and Veterans Affairs Department. Dr. Joshua Hare previously owned equity in Biscayne Pharmaceuticals, licensee of intellectual property used in this study. Biscayne Pharmaceuticals did not provide funding for this study. J.M.H. reported having a patent for cardiac cell-based therapy. He holds equity in Vestion Inc. and maintains a professional relationship with Vestion Inc. as a consultant and member of the Board of Directors and Scientific Advisory Board. J.M.H. is the Chief Scientific Officer, a compensated consultant and advisory board member for Longeveron, and holds equity in Longeveron. J.M.H. is also the co-inventor of intellectual property licensed to Longeveron. Longeveron LLC and Vestion Inc. did not participate in funding this work. Dr. Hare's relationships are disclosed to the University of Miami, and a management plan is in place. Dr. Konstantinos E. Hatzistergos discloses a relationship with Vestion Inc. that includes equity. K.E.H. is also the co-inventor of intellectual property licensed to Vestion. Ms. Valasaki and Drs. Wanschel, Salerno, Kuznetsov, Kurtenbach, Rodriguez, Balkan Dykxhoorn, declare no conflict of interests. 
Author Contributions. K.E.H. conceived, designed and performed the experiments and wrote the manuscript. A.C.B.A.W. performed experiments and assisted with manuscript writing. A.G.S., J.N.K., S.K., D.A.R., K.V., and D.D., performed and/or analyzed experiments. W.B., A.V.S. and J.M.H. conceived of the experimental design and assisted with manuscript writing.

Acknowledgements. This work was supported by the National Institutes of Health grants R01 HL107110, R01 HL137355, R01 HL084275 and 5UM HL113460; grants from the Starr Foundation and the Soffer Family Foundation (all awarded to J.M.H.); and by the São Paulo Research Foundation (FAPESP) grant\# 2016/01044-0 (A.G.S) and grant\# 2016/01746-4 (A.C.B.A.W). JMH is also supported by NIH grants 5UM1 HL113460, 1R01 HL134558, 5R01429 CA136387, and HHSN268201600012I, Department of Defense grant W81XWH-19PRMRP-CTA and the Soffer Family, Lipson Family, Marcus Family and Starr Foundations. 


\begin{abstract}
Differentiation and expansion of cardiomyocytes from human induced pluripotent stem cells is a poorly understood process, and the signals promoting differentiation of cardiomyocytes toward specific cell fates are not well defined. Here we show that the growth hormone-releasing hormone receptor (GHRH-R) signaling pathway controls this differentiation process and cell fate. Stimulation of hPSCs with GHRH promotes $\beta$-catenin stabilization through the GH/IGF1 axis. The presence of cell-surface GHRH-R differentiates between atrio-ventricular cardiomyocytes and non-cardiomyogenic cardiac cells, including coronary vascular, neuronal and pacemaker cells, such that selecting for GHRH-R allows for the elimination of pacemaker cells and derivation of a pure population of atrio-ventricular cardiomyocytes. Unlike SIRPA, cardiac GHRH-R identifies murine and human NKX2-5 $5^{+}$mocardial derivatives of both mesodermal and neuroectodermal lineages. Using RNA-seq to comprehensively characterize the GHRH-R signaling pathway, we identified that NKX2-5, a transcription factor involved in progenitor specification and differentiation into atrio-ventricular cardiomyocytes, is upregulated in response to GHRH. GHRH-R signaling also upregulated HIF-1 $\alpha$ via the PI3k/Akt/mTOR pathway to enhance NKX2-5 transcription and stimulate a Warburg-like effect in developing cardiomyocytes. Together these findings provide crucial insights into human stem cell differentiation and offer a translationally useful tool to optimize the development of pure cardiomyocyte preparations derived from induced pluripotent stem cells.
\end{abstract}

Keywords: cardiovascular progenitor, cardiomyocytes, iPSCs, GHRH, GHRH Receptor, NKX2-5, HIF-1 $\alpha$ 


\section{INTRODUCTION}

Cardiomyocytes are the muscle cells primarily responsible for heart contraction. These cells are highly differentiated, contractile, dense muscle cells, that are highly susceptible to injury or loss in large numbers following myocardial infarction $(1,2)$. The quest for replacement therapy has prompted substantial research aimed at developing in vitro differentiation protocols for converting pluripotent stem cells into cardiomyocyte-like cells $(3,4)$. For example, the 'pluripotent stem-cellderived cardiomyocyte' (hPSC-CM) protocol, achieves a stepwise differentiation of cells into all major cardiac mesoderm-derived lineages, using a combination of signaling cues based on similar signals that generate cardiomyocytes in vivo. The resulting hPSC-CMs express markers of atrioventricular cardiomyocytes, pacemaker cells, epicardial derivatives, coronary vascular cells, and, in vivo, can remuscularize infarcted regions of recipient hearts (5-8). However, a challenge for producing any cell type in vitro for transplantation purposes is the heterogeneity of the cells generated by directed differentiation, a situation that is particularly relevant for cardiomyocytes. For instance, current differentiation protocols also produce sino-atrial node hPSC-CMs, which have the potential to become dangerous artificial pacemakers in vivo (9). Indeed, malignant ventricular arrhythmias are evident in robust animal models of iPS directed remuscularization (8). To improve specificity, it is important to regulate the stages of differentiation and produce a pure population of cardiomyocytes.

Previous studies using cardiomyocyte differentiation protocols have identified stage specific cellsurface markers (early mesoderm- and cardiomyocyte-specific), that can facilitate the enrichment or depletion of specific cardiovascular sub-populations from hPSCs. Growth hormone-releasing hormone receptor (GHRHR) is a cell surface marker found on adult (10) and fetal (11) cardiomyocytes, suggesting GHRH/GHRHR signaling is crucial for cardiac development. However, whether and how this cell-surface molecule relates to the regulation of cardiac morphogenesis, and consequently the fate of cardiac cells in the developing embryo, are critical steps for advancing the directed differentiation of hPSCs (12) toward a treatment for myocardial infarction. 


\section{RESULTS}

\section{SC-Cardiomyocytes contain four major cell types}

Using an efficient hPSC-CM differentiation protocol (Fig. 1A), human pluripotent stem cells grown in clusters were differentiated in four stages using specific inducing factors to produce 'stem-cell cardiomyocytes' (SC-CM) some of which are atrio-ventricular cardiomyocytes. Progress and efficiency were measured using qPCR and immunofluorescence microscopy (Fig. 1B and 1C). On day 0 , progenitors formed a single heterogeneous population of cells. On day 2 , following 48 h of GSK3 inhibition by CHIR99021, to produce primitive streak-like mesodermal progenitors and then specify into cardiogenic mesoderm, we observed a transient up-regulation of $T$ ( $T$-box transcription factor Brachyury) and MESP1 which was sustained until day 5 . On days 2-5, after 2 days in the presence of the porcupine inhibitor IWP2, expression of OCT4 and NANOG, which (help) maintain stemness, was downregulated. We also observed expression of the key cardiomyocytic genes NKX2-5 (13) and ISL1 (14, 15), in progenitors on days 5-7, which predicted cardiac induction potential. Finally, 2 days after IWP2 removal, we observed a mixture of spontaneously beating atrio-ventricular cardiomyocytes and conduction system cells. This cardiac differentiation of hPSCs was performed according to previously described protocols (16).

To ensure cardiomyocyte differentiation, flow-cytometry analysis identifying PE cardiac specific marker cardiac troponin T (cTnT) and the NKX2-5-expressing cells and on d20 confirmed the efficiency of cardiomyocyte differentiation protocol, resulting in $99 \%$ and $81 \% \mathrm{CTnT}^{+}$and NKX2$5^{+}$cardiomyocytes, respectively (Fig. S1A). We next evaluated the percentage of $\mathrm{CTnT}^{+} / \mathrm{NKX}^{2} .5^{+}$ cells that also express GHRHR, which is exclusively expressed in NKX2-5 ${ }^{+}$cardiomyocytes and is absent in sinoatrial node (SAN)-like pacemaker cells (NKX2-5). Flow-cytometry analysis (Fig. $\mathrm{S} 1 \mathrm{~B}$, left panel) revealed that the vast majority of $\mathrm{CTnT}^{+} / \mathrm{NKX}_{2} .5^{+}$cells derived using the cardiomyocyte differentiation protocol were $\mathrm{GHRHR}^{+}$(Fig. S1B, right panels).

Interestingly, a small minority of the differentiating cells $(\sim 18 \%$ in the cardiomyocyte differentiation protocol, in the FACS example in Fig. S1A) were TnTpositive/Nkx2.5-negative. Such $\mathrm{TnT}^{+} / \mathrm{NKX} 2.5^{-}$cells, accounting for $18 \%$ of the cells in the cardiomyocyte differentiation protocol, were previously shown to represent sinoatrial node (SAN)-like pacemaker cells and may be responsible for the spontaneous automaticity of the generated engeniered heart tissue.

\section{Late SC-CM express GHRH-R along with NKX2-5}

Our analysis over the time-course of differentiation, identified that the expression of GHRHR and its ligand $G H R H$ did not occur coincident with $T$ and MESP1, but increased concurrently with NKX2-5 on days 5-9, compared to SIRPA (cell surface marker of all human cardiac myocyte 
subtypes, including conduction system cells) which was induced as early as day 5, prior to the onset of NKX2-5 (Fig. 1A-C). To investigate the relevance of our findings in vivo in the human heart, where we verified GHRH-R expression in developing human hearts at 16 weeks of gestation. Accordingly, GHRH-R expression was found to co-localize only with cardiac NKX2-5 cells (Fig. S2A), in a fashion similar to SIRPA expression (Fig. S2B). GHRH-R was not expressed in either NKX2-5 cells or in ISL1 ${ }^{+}$cell populations, the latter of which comprise neural crest (NC), second heart-field (SHF) and pacemaker cells.

To examine cardiac lineage-specific differences in ISL $1^{+}$cell population (14) in vivo, we employed Cre-Lox fate-mapping approaches in mice with the Mef2c-AHF-Cre;tdTomato and Wnt1-Cre2;tdTomato reporter alleles, which respectively mark anterior SHF (AHF) and NC cells, respectively, with the conditional fluorescence reporter tdTomato. Using this approach, we detected GHRH-R co-localization in NKX2-5 $5^{+}$cells that efficiently expressed tdTomato-AHF (right ventricle and interventricular septal wall cells), as well as in non-AHF cells (left ventricle, interventricular septal wall and atrial cells), as well as in non-AHF cells (left ventricle, interventricular septal wall and atrial cells) (Fig. S2C). GHRH-R was not expressed in NKX2-5cells, similar to the human hearts, regardless of the murine cells being tdTomato positive or negative. More importantly, we observed that conduction system cardiomyocytes in the sinus node, which descend from the posterior aspect of the SHF, do not express GHRH-R (Fig. S2D$\mathrm{G)}$.

More importantly, we observed that conduction system's cardiomyocytes in the sinus node, which descend from the posterior aspect of the SHF do not express GHRH-R (Fig. S2D-G).

Similarly, NC cells, including their ISL $1^{+}$cells in cardiac ganglia, do not express GHRH-R (Fig. S3 A-E). Remarkably, we found that tdTomato-NC cells gained GHRH-R expression upon differentiation into NKX2.5 $5^{+}$cells (Fig. S3 F-G).

SIRPA marks the cell surface of all human cardiac myocyte subtypes, including conduction system cells (17). To examine SIRPA expression compared to GHRH-R, we performed immunoreactivity experiments. However, as previously reported, the pattern of human cardiac SIRPA was not conserved in the murine heart and was undetectable in NKX2.5 cardiac cells (Fig. S3 H-I).

\section{Purification of cardiomyocytes}

Flow cytometry-based quantitative analysis on day 7, illustrates a virtually complete overlap of GHRH-R and NKX2-5 expression (Fig. 2A). Additionally, we show that at day 7 that purified GHRH-R immunoreactive cells, exhibited ted full capacity to differentiate into spontaneously 
beating cardiomyocytes (Movie S1). On day 9, we show that stained SC-CM expressing SIRPA do not express NKX2-5 (Fig. 2B). Using Short stature Homeobox 2 (SHOX2) expression to distinguish between atrioventricular $\left(\mathrm{NKX} 2-5^{+}\right)$and pacemaker cells $\left(\mathrm{NKX} 2-5^{-} / \mathrm{SHOX} 2^{+}\right.$), we discovered that GHRH-R is only expressed in atrio-ventricular cells and, therefore, can be used to distinguish them from pacemaker cardiomyocytes (Fig. 2B). Our data also revealed that cardiac differentiation of hPSCs generated a mixture of atrioventricular, pacemaker and neural crest cells (NC). Using the SOX10::GFP hPSC reporter line (18), we showed that NC cells do not express GHRH-R (Fig. 2B and 2C). Together, our analysis identifies GHRH-R as an atrio-ventricular SCCM surface marker (Fig. 2D).

\section{mTOR Pathway is necessary for GHRH-mediated NKX 2-5 expression}

We applied RNA sequencing (RNA-seq), to generate a deep understanding of GHRH-R signaling. To this end, we stimulated SC-CMs with $0 \mu \mathrm{M}, 0.15 \mu \mathrm{M}$ or $0.3 \mu \mathrm{M}$ of GHRH for 1 hour and identified 83 differentially expressed genes (DEGs) among the 3 groups (Fig. 3A).

Functional enrichment analysis identified specific signaling pathways, including upregulation of Activating Transcription Factor (ATF)-2, hypoxia inducible factor (HIF)-1 $\alpha$ and $-2 \alpha$, and erythropoietin (EPO) (Fig. 3B). GHRH signaling leads to a dose-dependent increase of transcription of NKX2-5 (Fig. 3C) and HIF-1 high mTOR pathway activity is recquired for GHRH NKX2-5 transcription, as the mTOR inhibitor LY294002 reduced rhGHRH induced NKX2-5 transcription (Fig. 3E).

We performed Western blotting to quantify specific components of the mTOR pathway, and observed that SC-CM stimulated with GHRH express significantly higher levels of pAKT, mTORC (Ser2448), mTORC (Ser2481) and phosphorylated tuberous sclerosis complex (p-TSC2) (Fig. $3 F)$. To examine the role of HIF-1a implication in the up-regulation of NKX2-5 in response to GHRH-R signaling, we employed CRISPR/spCas9 mediated knockout of HIF-1a in the hPSCs. In agreement with the importance of HIF-1 $\alpha$ in GHRH-R signaling HIF-1 $\alpha-K O$ hiPSC-CMs failed to increase NKX2-5 expression in response to rhGHRH (Fig. 3G). Genome-wide chromatin immunoprecipitation combined with high-throughput sequencing (ChIP-seq) studies revealed strong occupancy of NKX2-5 loci by HIF-1 $\alpha$ (Fig. 3H).

We compared HIF-1 $\alpha$ target genes identified by ChIP-seq with changes in RNA-seq and pathway activation following HIF-1 $\alpha$ knockout in hiPSCs-CMs (Fig. 3I). Accordingly, we identified HIF-1 $\alpha$ binding to hypoxia-response elements (Fig. 3l) and the mTOR pathway as the most significantly affected in the absence of HIF-1a (Fig. 3L). 


\section{$\beta$-catenin stabilization and signaling of GHRH-R in atrio-ventricular cardiomyocytes}

Single-cell resolution can monitor developmental trajectories from cells. Using a hPSC stably expressing a TCF/LEF:H2B-GFP construct, which is activated by $\beta$-catenin binding, (Fig. 4A), we sought to determine $\beta$-catenin levels at single-cell resolution via the expression of a $H 2 B-G F P$ reporter placed under the control of six copies of a TCF/LEF responsive element and an $h s p 68$ minimal promoter. $\beta$-catenin, a temporal transcriptional factor and regulator of stage-specific differentiation and proliferation, is expressed by numerous embryonic cells including pre-cardiac progenitors, cardiac precursor cells, and differentiated cardiomyocytes. Differentiation of hPSCs into primitive streak-like cells in response to CHIR99021-mediated $\beta$-catenin stabilization. On day 2, we observed a robust H2B-GFP expression (Fig. 4A) as well as expression of somatostatin (SST; GHRH antagonist) (Fig. 4B). The initiation of cardiogenic differentiation is a major event during stage 2 . On day 4 , primitive streak-like cells exhibited a sharp reduction in H2B-GFP expression representing the antagonism of $\beta$-catenin stabilization - marked by SST downregulation and GH1, IGF1 upregulation (Fig. 4B). On day 5, the emergence of a minor pool of cells with mild H2B-GFP expression reflect the termination of $\beta$-catenin antagonism to promote differentiation of progenitors into cardiac-like NKX2-5 $5^{+}$precursors - gene expression gradient shows SST recovery and GH1, IGF1 repression (Fig. 4B).

By immunostaining (Fig. 4C), we verified that H2B-GFP(+) cells co-localized (yellow) with GHRH$\mathrm{R}$ in NKX2-5+ myocardial precursors (Upper panel), which suggesting that there is a relationship between the $\mathrm{GHRH}-\mathrm{R}$ and $\beta$-catenin signaling. Upon stimulation with $\mathrm{GHRH}$, we also observed the emergence of $\mathrm{H}_{2} \mathrm{~B}-\mathrm{GFP}^{+}$cells (Fig. 4D), which also exhibit an elevated level of Akt activity (Fig. 4E).

\section{GHRH-R removal reduces NKX2-5 transcription}

We sought to determine the GHRH-R function and signaling in cardiogenesis using a CRISPR/spCas9 functional genomic approach. The human GHRH receptor gene (GHRHR) is located on chromosome 7p14 and consists of 13 exons, with the transcription start site located in exon 1 and the stop codon in exon 13. Exons 1-4 encode the extracellular signaling domain, exons 5-12 the transmembrane domain and exons 12-13 the intracellular domains of the receptor. Moreover, exon 1 encodes the first 18 amino acids of GHRH-R which translates as the signal peptide; mutations $(18,19)$ or deletion $(20)$ of which lead to GHRH-R loss-of-function and the development of isolated growth hormone deficiency (IGHD) in mice and humans. Accordingly, to knock-out the GHRH-R signaling peptide in hPSCs we generated a stable cell line expressing a tetracycline-inducible spCas9 and a gRNA spanning the ATG start sites of exon 1, with no 
predictable off target effects (Fig. 5A-B). DNA mismatch cleavage detection analysis illustrated $\sim 71 \%$ efficiency in genome editing of the targeted region in response to 10-day treatment with doxycycline (Fig. 5B). As GHRHR is silenced in undifferentiated hPSCs (Fig. 1B), the resulting clones were indistinguishable from wildtype hPSCs (Fig. 5C). Figure 5D depicts Western blot analysis of GSK-3 $\beta$ and Akt activity in hPSC wild type or knockout to GHRHR treated with GHRH, somatostatin (SST) or combination of both (GHRH+S), confirming the dependence of GSK-3 $\beta$ and Akt expression on GHRH-R signaling.

\section{Sustained expression of GHRH-R enhances atrio-ventricular differentiation}

We overexpressed GHRH-R in undifferentiated iPSCs. Lentiviral overexpression of GHRHR resulted in an 8- fold increase in the proportion of GHRHR expression compared with controls (Fig. 6A-B). Consistent with a role of GRHR-R controlling NKX2-5 expression, GHRH-R overexpression leads to a significant upregulation of NKX2-5 levels (Fig. 6C). Beyond GHRH-R expression, we explored ways of enriching for atrio-ventricular cardiomyocytes.

\section{Activation of HIF-1 $\alpha$ and Hexokinase pathway in cultured hPSC-CMs.}

HIF-1 $\alpha$ is a central regulator of metabolism and promotes glycolysis at the expense of oxidative phosphorylation (21-25). We identified specific signaling pathways that are upregulated upon GHRH-R stimulation, including upregulation of hypoxia inducible factor (HIF)-1 $\alpha$ and $-2 \alpha$; To test for a potential role for this pathway in controlling the energy substrate selection, we measured the mRNA levels of direct and indirect downstream targets of activated HIF-1 $\alpha$ and examined mitochondrial function. Among these transcripts, we found that the glycolytic enzyme hexokinase (HK)-1 and -2 are upregulated in the hPSC-CMs overexpressing GHRH-R (Fig. 7A). Other transcripts involved in cellular metabolism were not significantly different between the groups. The extracellular acidification rate (ECAR) indicates that glycolysis is enhanced following $\mathrm{GHRH}$ treatment. Schematic realtime analysis of glycolysis showed that following GHRH treatment PSC$\mathrm{CM}$ exhibited a higher maximum glycolysis induced by oligomycin in the presence of glucose (Fig. 7F). Quantitative analysis showed no significant differences during the basal stage between groups (Fig. 7G). In the presence of glucose, the glycolytic rate of PSC-CM treated with GHRH increased compared with the basal stage with treated cells exhibiting a higher glycolytic rate than control (Fig. 7H). The glycolytic capacity of GHRH treated PSC-CM was higher than control PSCCMs (Fig. 7I). However, in contrast to glycolytic rate, glycolytic reserve was the lower in control PSC-CMs (Fig. 7J). Finally, there is no change in the mitochondrial OCR after GHRH treatment (OCR, Fig. 7B). In addition, the ATP production was significantly higher in hPSC-CMs upon GHRH treatment compared to control hPSC-CMs (Fig. 7D). These results support our findings 
that upon exposure to $\mathrm{GHRH}$, hPSC-CMs are metabolically reliant on glycolysis rather than oxidative phosphorylation and furthermore, that they generate more ATP compared to hPSC-CMs not stimulated with GHRH.

\section{hPSC-CMs overexpressing GHRHR lacks metabolic and functional maturation.}

Prior reports have suggested that the mammalian embryonic heart depends on glycolysis for ATP production whereas the adult heart switches primarily to oxidative phosphorylation to produce energy. Since our results showed that hPSC-CMs upon GHRH activation have a high glycolytic rate that is similar to embryonic and postnatal hearts, we examined to examined mitochondrial structural features in the iPSC-CMs with transmission electron microscopy. Figure S4A shows a photomicrography of a typical iPSC-CM mitochondria. Significantly shorter mitochondria (Fig. S4B), with no changes in mitochondrial number, were observed in iPSC-CMs overexpressing GHRH-R, i.e., these cells have the same number, but smaller mitochondria. Using mitotracker green staining and quantitative flow cytometry, we observed that hPSC-CMs overexpressing GHRH-R have significantly lower median mitrotracker intensities compared to control hPSC-CMs (Fig. S4D), indicating reduced mitochondrial density, in agreement with the TEM observations. We quantified sarcomere length to assess the impact of GHRH on the structural maturation of hPSC-CMs; however, we did not observe any changes between groups (Fig. S4C). Together, these results show that GHRH responsiveness produces hPSC-CMs that resemble immature embryonic and neonatal cardiomyocytes with respect to their energy substrate utilization, mitochondrial content, and structure.

\section{DISCUSSION}

Atrio-ventricular cardiomyocytes, are an ideal candidate for cell-based therapies to remuscularize the heart in patients suffering from a myocardial infarction. Nonetheless, directed differentiation protocols for atrio-ventricular cardiomyocytes also generate pacemaker cardiomyocytes. In this study, we identified GHRH-R as a surface marker of atrio-ventricular cardiomyocytes and use RNA-seq experiments to comprehensively characterize its signaling pathways using RNA-seq. Here we found that NKX2-5, a transcription factor involved in progenitor specification and differentiation into atrio-ventricular cardiomyocytes is an effector of the GHRH-R signaling pathway. GHRH-R signaling upregulates HIF-1a via PI3k/Akt/mTOR pathway to enhance NKX25 transcription. Nonetheless, antagonism of GHRH/GHRH-R signaling in heart failure exerts cardioprotective and reparative effects partly via stimulation of the PI3K-Akt pathway (26-30). 
Our analysis determined the cardiac lineage-specific differences in vivo. We identified that ISL-1 positive cell populations, NC, and SHF and pacemaker cells, do not express GHRHR. However, we showed that NC cells that are NKX2-5, acquire GHRH-R expression upon differentiation into

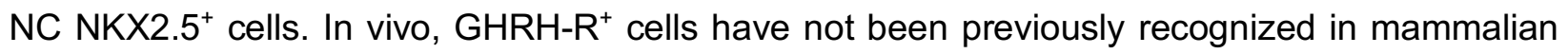
hearts. Exploiting our in vitro findings that expression of GHRH-R and its ligand GHRH increase coincidently with NKX2.5 expression, we uncovered GHRH-R is co-localized strictly on NKX2-5 ${ }^{+}$ cardiac cells in murine and human developing hearts.

The stepwise synchronous differentiation of millions of cells provides an opportunity to study developmental processes. Dynamically, different cardiomyocyte subtypes in the heart derive from distinct mesodermal populations that are induced in a defined temporal pattern. The temporal transcription factor $\beta$-catenin drives pre-cardiac progenitor compartmentalization, whereas the antagonism of $\beta$-catenin stabilization increases the preponderance of cardiac-like precursor cells and subsequently differentiated cardiomyocytes (31-33). The present study identified that GHRH/GHRH-R signaling in hPSCs-NKX2-5 mediates $\beta$-catenin stabilization through the GH/IGF1 axis. Specifically, exposure of cells at the mesodermal stage to insulin or IGF-1 induces the PI3K-Akt pathway, which in turn stabilizes $\beta$-catenin by phosphorylating and inactivating its cytoplasmic inhibitor GSK3 $\beta$ kinase (34-37). The importance of this mechanism in cardiomyocyte generation is reflected in the strong inhibitory effects of exogenous insulin on cardiogenic differentiation of hPSCs $(35,37)$. Upstream of this pathway lies the GH/IGF-1 axis, which itself is positively regulated via two other hormones, Ghrelin and $\mathrm{GHRH}$, and negatively regulated by SST (38). GHRH functions by binding to its G-protein coupled receptor, GHRH-R, but it's role in human cardiovascular development and pathophysiology is unclear, and as our study suggests, needs additional study. Mutations in GHRH-R lead to IGHD, the manifestations of which include reduced heart size and elevated cardiovascular risk factors, including impaired glucose tolerance and increased lipid profiles and systolic blood pressure $(39,40)$. Our data suggest a relationship between the GHRH-R and $\beta$-catenin signaling, and that by alternating this relationshipwe can manipulate the outcome of this differentiation.

We show that SC-CM maintain their identity under extended growth in culture in the absence of signaling modulators. Dynamic genes include several markers of cardiomyocytes maturation (SIRPA/Troponin). Furthermore, the identity of heterogeneous pool of cells has previously show: markers of atrio-ventricular cardiomyocytes, pacemaker cells, epicardial derivatives and coronary vascular cells. In the context of transplantation, these cells may improve cardiac function; however, as demonstrated with hPSC-CM in non-human primate $(41,42)$ and swine $(43)$ models, 
they can have arrhythmogenic potential. We show that progenitors that progress towards atrioventricular cardiomyocytes are GHRHR+NKX2-5+. Compared to SIRPA (SIRPa), cardiac GHRH$\mathrm{R}$ identifies murine and human NKX2-5+ myocardial derivatives of both mesodermal and neuroectodermal lineages. Moreover, GHRH-R distinguishes the cell-surface of cardiomyogenic from non-cardiomyogenic cardiac cells, such as coronary vascular, neuronal and pacemaker cells.

Compared to human adult cardiomyocytes, hPSC-CMs are relatively immature both morphologically and functionally, lacking normal levels of binucleation, well-developed sarcomeres, fast electrical conductivity, and oxidative metabolism (44). We, therefore, sought to investigate the mechanism underlying metabolic substrate selection of hPSC-CMs upon GHRH to determine whether this metabolic pathway controlled myocardial maturation.

We observed increases in non-mitochondrial OCR, ATP production as well as in cellular hexokinase activity levels in hPSC-CMs stimulated with GHRH. Hexokinase is a key enzyme in glycolysis that converts glucose into glucose-6-phosphate and its activity levels are a surrogate for glycolysis rates (45-47). These results critically show that GHRH keeps CM metabolism at glycolytic pathways and are aligned with findings described by Xiang $P$ et al., (48) using GHRH agonist MR409. Several studies have demonstrated that the embryonic and neonatal mammalian heart depends on aerobic glycolysis as the principal energy production pathway (49). Furthermore, we showed that HIF-1a is activated in hPSC-CMs following exposure to GHRH. HIF-1 1 signaling in cancer cells increase glycolysis and lactate fermentation rates (50-52) while deactivating oxidative phosphorylation (52). HIF-1a is a master transcriptional regulator of cellular metabolism (53) and its expression is crucial for early fetal cardiac development $(54,55)$. Recent studies have further suggested that activation of the HIF-1a pathway is required for the maintenance of a myocardial proliferative state during early embryonic development (25). The expression and activity of HIF-1 $\alpha$ in hPSC-CMs cultured in the presence of GHRH is similar to its reported expression during early fetal development. Moreover, expression of GHRH-R during the mid-gestation stage of cardiac development stabilizes HIF-1a protein and prevents the metabolic shift from glycolytic to oxidative pathway, but with no but with no effect on cardiac maturation (25).

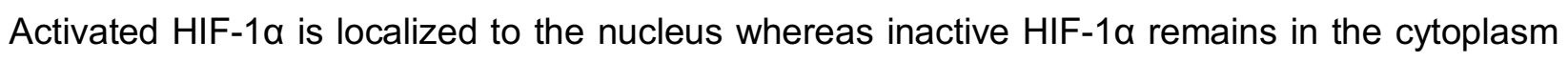
where it is rapidly turned over $(54,56,57)$. Activation of HIF-1 $\alpha$ further results in increased HIF1 $\alpha$ nuclear localization, consistent with our results of increased HIF-1 $\alpha$ DNA binding activity (23, 58-60). 
In summary we identified GHRH-R as a cell surface marker of atrio-ventricular cardiomyocytes, that enables selection for eliminating pacemaker cells and the derivation of a pure population of atrio-ventricular cells. Additionally, we demonstrated a method that enriches atrio-ventricular cardiomyocytes, providing a resource for future development of differentiation protocols and suggests a mechanism by which cell fate is modulated by signaling pathways, bioenergetics and energy substrate utilization all guided by receptor expression patterns and downstream signaling activities.

\section{REFERENCES}

1. Laflamme MA, and Murry CE. Regenerating the heart. Nat Biotechnol. 2005;23(7):84556.

2. Murry CE, Reinecke H, and Pabon LM. Regeneration gaps: observations on stem cells and cardiac repair. J Am Coll Cardiol. 2006;47(9):1777-85.

3. Lee JH, Protze SI, Laksman Z, Backx PH, and Keller GM. Human Pluripotent Stem CellDerived Atrial and Ventricular Cardiomyocytes Develop from Distinct Mesoderm Populations. Cell Stem Cell. 2017;21(2):179-94 e4.

4. Protze SI, Liu J, Nussinovitch U, Ohana L, Backx PH, Gepstein L, et al. Sinoatrial node cardiomyocytes derived from human pluripotent cells function as a biological pacemaker. Nat Biotechnol. 2017;35(1):56-68.

5. Caspi O, Huber I, Kehat I, Habib M, Arbel G, Gepstein A, et al. Transplantation of human embryonic stem cell-derived cardiomyocytes improves myocardial performance in infarcted rat hearts. J Am Coll Cardiol. 2007;50(19):1884-93.

6. Chong JJ, and Murry CE. Cardiac regeneration using pluripotent stem cells--progression to large animal models. Stem Cell Res. 2014;13(3 Pt B):654-65. 
7. Laflamme MA, Chen KY, Naumova AV, Muskheli V, Fugate JA, Dupras SK, et al. Cardiomyocytes derived from human embryonic stem cells in pro-survival factors enhance function of infarcted rat hearts. Nat Biotechnol. 2007;25(9):1015-24.

8. Shiba Y, Gomibuchi T, Seto T, Wada Y, Ichimura H, Tanaka Y, et al. Allogeneic transplantation of iPS cell-derived cardiomyocytes regenerates primate hearts. Nature. 2016;538(7625):388-91.

9. Burridge PW, Matsa E, Shukla P, Lin ZC, Churko JM, Ebert AD, et al. Chemically defined generation of human cardiomyocytes. Nat Methods. 2014;11(8):855-60.

10. Alagiakrishnan K, Banach M, Jones LG, Datta S, Ahmed A, and Aronow WS. Update on diastolic heart failure or heart failure with preserved ejection fraction in the older adults. Annals of medicine. 2013;45(1):37-50.

11. Christodoulou C, Schally AV, Chatzistamou I, Kondi-Pafiti A, Lamnissou K, Kouloheri S, et al. Expression of growth hormone-releasing hormone (GHRH) and splice variant of GHRH receptors in normal mouse tissues. Regulatory peptides. 2006;136(1-3):105-8.

12. Skelton RJP, Kamp TJ, Elliott DA, and Ardehali R. Biomarkers of Human Pluripotent Stem Cell-Derived Cardiac Lineages. Trends Mol Med. 2017;23(7):651-68.

13. Lints TJ, Parsons LM, Hartley L, Lyons I, and Harvey RP. Nkx-2.5: a novel murine homeobox gene expressed in early heart progenitor cells and their myogenic descendants. Development. 1993;119(3):969.

14. Bu L, Jiang X, Martin-Puig S, Caron L, Zhu S, Shao Y, et al. Human ISL1 heart progenitors generate diverse multipotent cardiovascular cell lineages. Nature. 2009;460(7251):1137.

15. Qyang Y, Martin-Puig S, Chiravuri M, Chen S, Xu H, Bu L, et al. The renewal and differentiation of IsI1+ cardiovascular progenitors are controlled by a Wnt/beta-catenin pathway. Cell Stem Cell. 2007;1(2):165-79. 
16. Lian X, Hsiao C, Wilson G, Zhu K, Hazeltine LB, Azarin SM, et al. Robust cardiomyocyte differentiation from human pluripotent stem cells via temporal modulation of canonical Wnt signaling. Proc Natl Acad Sci U S A. 2012;109(27):E1848-57.

17. Dubois NC, Craft AM, Sharma P, Elliott DA, Stanley EG, Elefanty AG, et al. SIRPA is a specific cell-surface marker for isolating cardiomyocytes derived from human pluripotent stem cells. Nat Biotechnol. 2011;29(11):1011-8.

18. de Graaff LC, Argente J, Veenma DC, Herrebout MA, Friesema EC, Uitterlinden AG, et al. Genetic screening of a Dutch population with isolated GH deficiency (IGHD). Clin Endocrinol (Oxf). 2009;70(5):742-50.

19. Godi M, Mellone S, Petri A, Arrigo T, Bardelli C, Corrado L, et al. A recurrent signal peptide mutation in the growth hormone releasing hormone receptor with defective translocation to the cell surface and isolated growth hormone deficiency. J Clin Endocrinol Metab. 2009;94(10):3939-47.

20. Sundralingam T, Tennekoon KH, de Silva S, De Silva S, and Hewage AS. Pathogenic and likely pathogenic genetic alterations and polymorphisms in growth hormone gene (GH1) and growth hormone releasing hormone receptor gene (GHRHR) in a cohort of isolated growth hormone deficient (IGHD) children in Sri Lanka. Growth Horm IGF Res. 2017;36:22-9.

21. Dengler VL, Galbraith M, and Espinosa JM. Transcriptional regulation by hypoxia inducible factors. Crit Rev Biochem Mol Biol. 2014;49(1):1-15.

22. Kim JW, Tchernyshyov I, Semenza GL, and Dang CV. HIF-1-mediated expression of pyruvate dehydrogenase kinase: a metabolic switch required for cellular adaptation to hypoxia. Cell Metab. 2006;3(3):177-85.

23. Semenza GL. Hypoxia-inducible factor 1: regulator of mitochondrial metabolism and mediator of ischemic preconditioning. Biochim Biophys Acta. 2011;1813(7):1263-8. 
24. Krishnan J, Ahuja P, Bodenmann S, Knapik D, Perriard E, Krek W, et al. Essential role of developmentally activated hypoxia-inducible factor 1alpha for cardiac morphogenesis and function. Circ Res. 2008;103(10):1139-46.

25. Menendez-Montes I, Escobar B, Palacios B, Gomez MJ, Izquierdo-Garcia JL, Flores L, et al. Myocardial VHL-HIF Signaling Controls an Embryonic Metabolic Switch Essential for Cardiac Maturation. Dev Cell. 2016;39(6):724-39.

26. Florea V, Majid SS, Kanashiro-Takeuchi RM, Cai RZ, Block NL, Schally AV, et al. Agonists of growth hormone-releasing hormone stimulate self-renewal of cardiac stem cells and promote their survival. Proc Natl Acad Sci U S A. 2014;111(48):17260-5.

27. Gesmundo I, Miragoli M, Carullo P, Trovato L, Larcher V, Di Pasquale E, et al. Growth hormone-releasing hormone attenuates cardiac hypertrophy and improves heart function in pressure overload-induced heart failure. Proc Natl Acad Sci U S A. 2017;114(45):12033-8.

28. Granata R, Trovato L, Gallo MP, Destefanis S, Settanni F, Scarlatti F, et al. Growth hormone-releasing hormone promotes survival of cardiac myocytes in vitro and protects against ischaemia-reperfusion injury in rat heart. Cardiovasc Res. 2009;83(2):303-12.

29. Kanashiro-Takeuchi RM, Takeuchi LM, Rick FG, Dulce R, Treuer AV, Florea V, et al. Activation of growth hormone releasing hormone $(\mathrm{GHRH})$ receptor stimulates cardiac reverse remodeling after myocardial infarction (MI). Proc Natl Acad Sci U S A. 2012;109(2):559-63.

30. Kanashiro-Takeuchi RM, Tziomalos K, Takeuchi LM, Treuer AV, Lamirault G, Dulce R, et al. Cardioprotective effects of growth hormone-releasing hormone agonist after myocardial infarction. Proc Natl Acad Sci U S A. 2010;107(6):2604-9.

31. Mazzotta S, Neves C, Bonner RJ, Bernardo AS, Docherty K, and Hoppler S. Distinctive Roles of Canonical and Noncanonical Wnt Signaling in Human Embryonic Cardiomyocyte Development. Stem Cell Reports. 2016;7(4):764-76. 
32. Ueno S, Weidinger G, Osugi T, Kohn AD, Golob JL, Pabon L, et al. Biphasic role for Wnt/beta-catenin signaling in cardiac specification in zebrafish and embryonic stem cells. Proc Natl Acad Sci U S A. 2007;104(23):9685-90.

33. Naito AT, Shiojima I, Akazawa H, Hidaka K, Morisaki T, Kikuchi A, et al. Developmental stage-specific biphasic roles of Wnt/beta-catenin signaling in cardiomyogenesis and hematopoiesis. Proc Natl Acad Sci U S A. 2006;103(52):19812-7.

34. Cross DA, Alessi DR, Cohen P, Andjelkovich M, and Hemmings BA. Inhibition of glycogen synthase kinase-3 by insulin mediated by protein kinase B. Nature. 1995;378(6559):7859.

35. Freund C, Ward-van Oostwaard D, Monshouwer-Kloots J, van den Brink S, van Rooijen $M, X u X$, et al. Insulin redirects differentiation from cardiogenic mesoderm and endoderm to neuroectoderm in differentiating human embryonic stem cells. Stem Cells. 2008;26(3):724-33.

36. Xin M, Kim Y, Sutherland LB, Qi X, McAnally J, Schwartz RJ, et al. Regulation of insulinlike growth factor signaling by Yap governs cardiomyocyte proliferation and embryonic heart size. Sci Signal. 2011;4(196):ra70.

37. Lian X, Zhang J, Zhu K, Kamp TJ, and Palecek SP. Insulin inhibits cardiac mesoderm, not mesendoderm, formation during cardiac differentiation of human pluripotent stem cells and modulation of canonical Wnt signaling can rescue this inhibition. Stem Cells. 2013;31(3):447-57.

38. Barabutis N, and Schally AV. Growth hormone-releasing hormone: extrapituitary effects in physiology and pathology. Cell Cycle. 2010;9(20):4110-6.

39. Oliveira CR, Salvatori R, Barreto-Filho JA, Rocha IE, Mari A, Pereira RM, et al. Insulin sensitivity and beta-cell function in adults with lifetime, untreated isolated growth hormone deficiency. J Clin Endocrinol Metab. 2012;97(3):1013-9. 
40. Barreto-Filho JA, Alcantara MR, Salvatori R, Barreto MA, Sousa AC, Bastos V, et al. Familial isolated growth hormone deficiency is associated with increased systolic blood pressure, central obesity, and dyslipidemia. J Clin Endocrinol Metab. 2002;87(5):201823.

41. Chong JJ, Yang X, Don CW, Minami E, Liu YW, Weyers JJ, et al. Human embryonic-stemcell-derived cardiomyocytes regenerate non-human primate hearts. Nature. 2014;510(7504):273-7.

42. Liu YW, Chen B, Yang X, Fugate JA, Kalucki FA, Futakuchi-Tsuchida A, et al. Human embryonic stem cell-derived cardiomyocytes restore function in infarcted hearts of nonhuman primates. Nat Biotechnol. 2018;36(7):597-605.

43. Romagnuolo R, Masoudpour H, Porta-Sanchez A, Qiang B, Barry J, Laskary A, et al. Human Embryonic Stem Cell-Derived Cardiomyocytes Regenerate the Infarcted Pig Heart but Induce Ventricular Tachyarrhythmias. Stem Cell Reports. 2019;12(5):967-81.

44. Yang X, Pabon L, and Murry CE. Engineering adolescence: maturation of human pluripotent stem cell-derived cardiomyocytes. Circ Res. 2014;114(3):511-23.

45. Monge C, Beraud N, Tepp K, Pelloux S, Chahboun S, Kaambre T, et al. Comparative analysis of the bioenergetics of adult cardiomyocytes and nonbeating HL-1 cells: respiratory chain activities, glycolytic enzyme profiles, and metabolic fluxes. Can J Physiol Pharmacol. 2009;87(4):318-26.

46. Calmettes G, John SA, Weiss JN, and Ribalet B. Hexokinase-mitochondrial interactions regulate glucose metabolism differentially in adult and neonatal cardiac myocytes. J Gen Physiol. 2013;142(4):425-36.

47. Roberts DJ, Tan-Sah VP, Ding EY, Smith JM, and Miyamoto S. Hexokinase-II positively regulates glucose starvation-induced autophagy through TORC1 inhibition. Mol Cell. 2014;53(4):521-33. 
48. Xiang $P$, Jing W, Lin Y, Liu Q, Shen J, Hu X, et al. Improvement of cardiac and systemic function in old mice by agonist of growth hormone-releasing hormone. J Cell Physiol. 2021.

49. Lopaschuk GD, and Jaswal JS. Energy metabolic phenotype of the cardiomyocyte during development, differentiation, and postnatal maturation. J Cardiovasc Pharmacol. 2010;56(2):130-40.

50. Courtnay R, Ngo DC, Malik N, Ververis K, Tortorella SM, and Karagiannis TC. Cancer metabolism and the Warburg effect: the role of HIF-1 and PI3K. Mol Biol Rep. 2015;42(4):841-51.

51. Sutendra G, Dromparis P, Kinnaird A, Stenson TH, Haromy A, Parker JM, et al. Mitochondrial activation by inhibition of PDKII suppresses HIF1a signaling and angiogenesis in cancer. Oncogene. 2013;32(13):1638-50.

52. Brand A, Singer K, Koehl GE, Kolitzus M, Schoenhammer G, Thiel A, et al. LDHAAssociated Lactic Acid Production Blunts Tumor Immunosurveillance by T and NK Cells. Cell Metab. 2016;24(5):657-71.

53. Semenza GL. HIF-1: mediator of physiological and pathophysiological responses to hypoxia. J Appl Physiol (1985). 2000;88(4):1474-80.

54. Yamashita $T$, Ohneda O, Nagano M, lemitsu M, Makino $\mathrm{Y}$, Tanaka $\mathrm{H}$, et al. Abnormal heart development and lung remodeling in mice lacking the hypoxia-inducible factorrelated basic helix-loop-helix PAS protein NEPAS. Mol Cell Biol. 2008;28(4):1285-97.

55. Dunwoodie SL. The role of hypoxia in development of the Mammalian embryo. Dev Cell. 2009;17(6):755-73.

56. Huang LE, Arany Z, Livingston DM, and Bunn HF. Activation of hypoxia-inducible transcription factor depends primarily upon redox-sensitive stabilization of its alpha subunit. J Biol Chem. 1996;271(50):32253-9. 
57. Kallio PJ, Pongratz I, Gradin K, McGuire J, and Poellinger L. Activation of hypoxiainducible factor 1alpha: posttranscriptional regulation and conformational change by recruitment of the Arnt transcription factor. Proc Natl Acad Sci U S A. 1997;94(11):566772.

58. Kelly TJ, Souza AL, Clish CB, and Puigserver P. A hypoxia-induced positive feedback loop promotes hypoxia-inducible factor 1alpha stability through miR-210 suppression of glycerol-3-phosphate dehydrogenase 1-like. Mol Cell Biol. 2011;31(13):2696-706.

59. Qutub AA, and Popel AS. Three autocrine feedback loops determine HIF1 alpha expression in chronic hypoxia. Biochim Biophys Acta. 2007;1773(10):1511-25.

60. Prabhakar NR, and Semenza GL. Adaptive and maladaptive cardiorespiratory responses to continuous and intermittent hypoxia mediated by hypoxia-inducible factors 1 and 2 . Physiol Rev. 2012;92(3):967-1003. 


\section{A Primitive streak \\ Days 0-2 CHIR99021 Insulin-free}

\section{B}

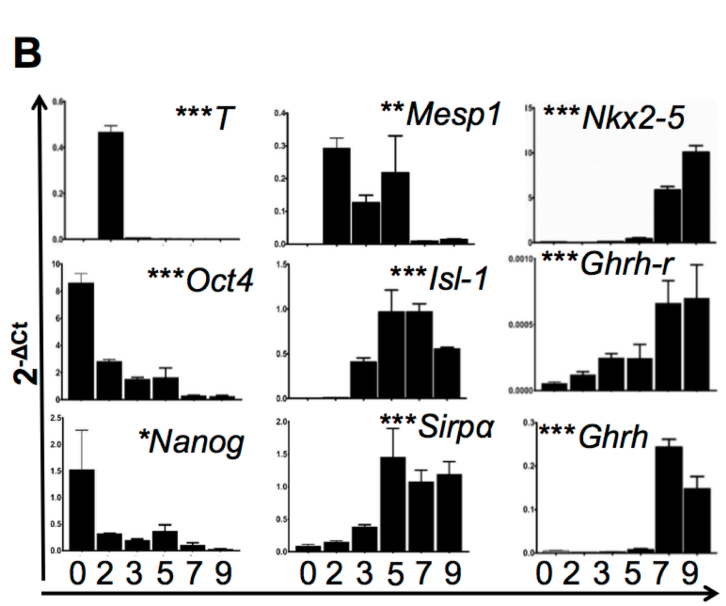

Days of Differentiation

\begin{abstract}
Precardiac progenitors

Days 3-5

IWP2

Insulin-free
\end{abstract}

Cardiac

Precursors

Days 5-7

Insulin-free
Days 8-10

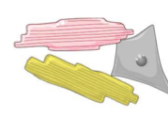

AtrioVentricular

+ pacemaker

cardiomyocytes

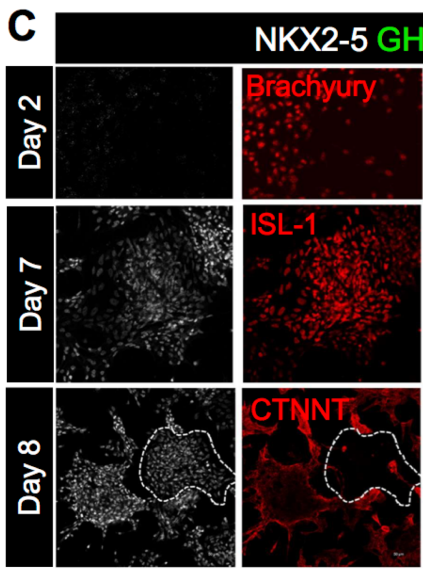

\section{NKX2-5 GHRH-R DAPI}

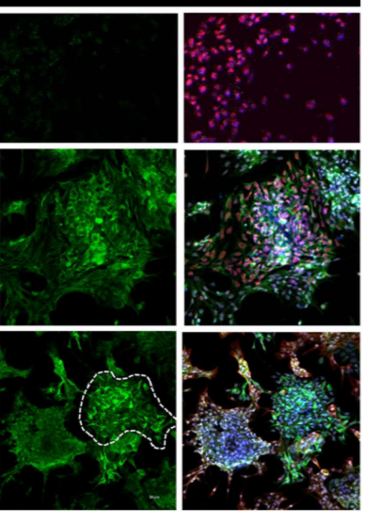

Fig.1. Differentiation and characterization of the human inducible pluripotent stem cells derived cardiomyocytes. A, Schematic representation of the human inducible pluripotent stem cells (hiPSC) differentiation protocols used to derive atrioventricular and pacemaker cardiomyocytes. B, qRT-PCR analysis of nascent mesoderm (T - Brachyury), cardiac mesoderm (Mesp-1, Isl-1), and cardiac progenitor (NKX2-5) genes; pluripotency (Oct4 and Nanog) and cellsurface cardiomyocyte marker SIRPA (SIRPa); GHRH and GHRH-R during cardiomyocyte differentiation. Expression values were normalized to GAPDH C, Immunofluorescence for NKX25, GHRH-R, Dapi and Brachyury on Day 2; and ISL-1 on Day 7; and CTNNT on Day 8. Error bars represent SEM. ${ }^{*} p<0.05,{ }^{* *} p<0.01,{ }^{* * *} p<0.001$; unpaired two-tailed $t$ test. 


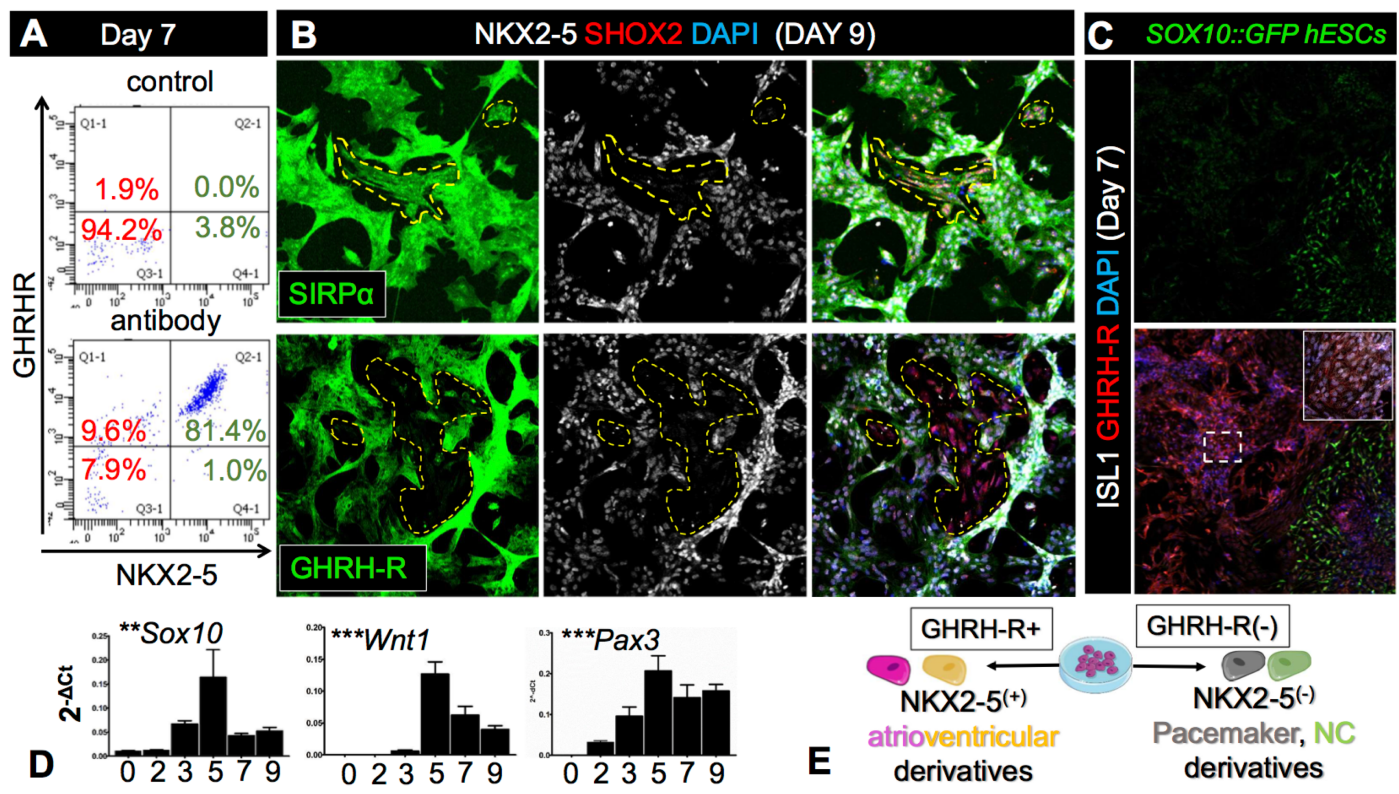

Fig.2. Expression of GHRH-R as a surface marker during human cardiomyocyte differentiation. A, Representative fluorescence-activated cell sorter (FACS) analysis of the overlap of GHRH-R and NKX2-5 at Day 7 of cardiomyocyte differentiation. B, Coimmunohistochemistry for NKX2-5, SHOX2, Dapi and SIRPA (SIRPa) and GHRH-R during cardiomyocyte differentiation on Day 9. Note that GHRH-R is only expressed in atrio-ventricular cells and not in pacemaker cells. C, Co-immunohistochemistry for ISL1, GHRH-R and Dapi on day 7 assembly by SOX10::GFP hPSC reporter line. D, qRT-PCR analysis of Expression values were normalized to GAPDH. E, Network model of GHRH-R+ as an atrio-ventricular SM-CM surface marker during cardiomyocytes differentiation. 


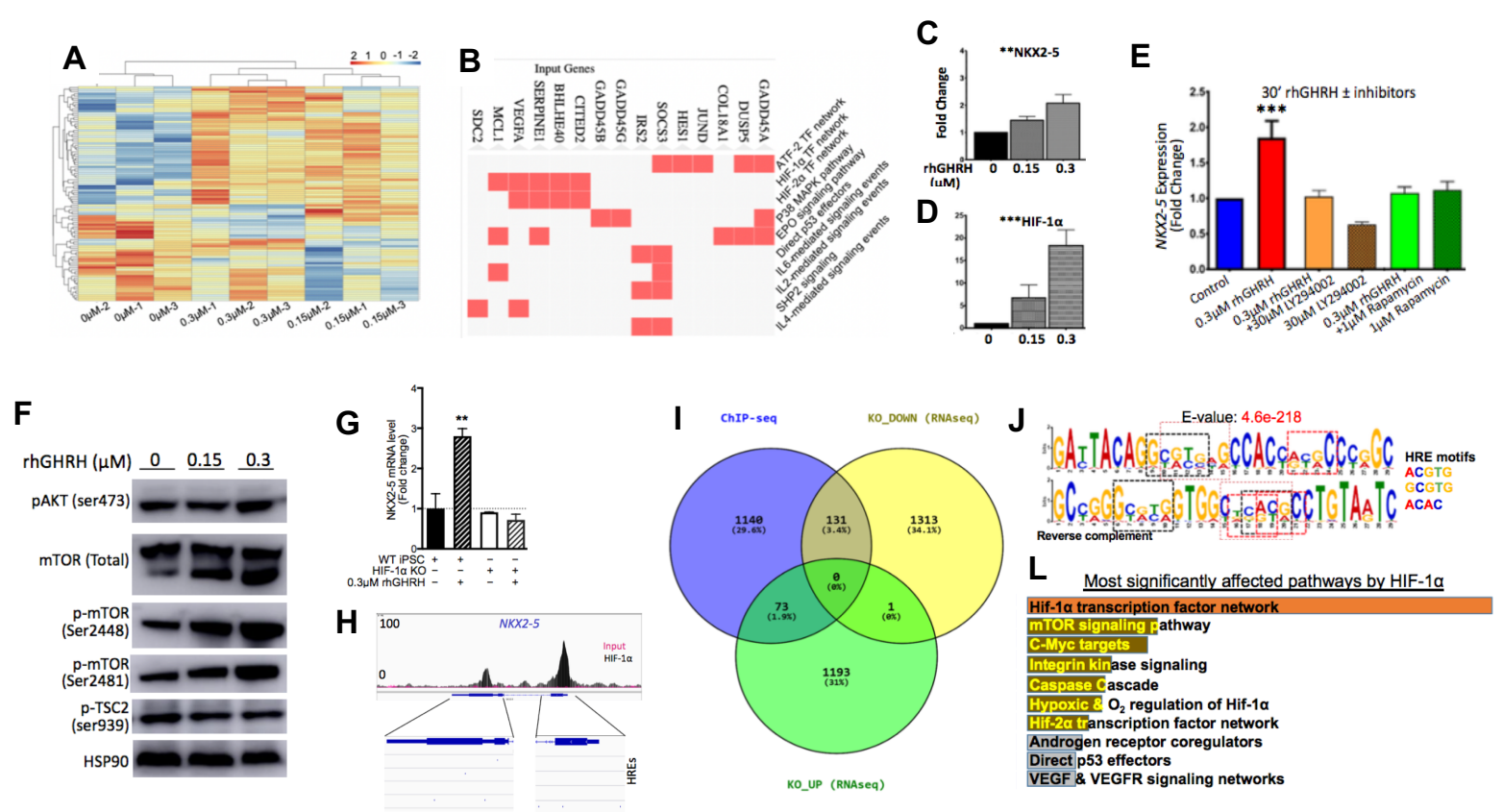

Fig.3. mTOR Pathway is necessary for GHRH-mediated NKX 2-5 expression. A, Heatmap showing that different concentration $(0,0.15,0.3 \mu \mathrm{M})$ of $\mathrm{GHRH}-\mathrm{R}$ changes corresponding to the 1434 transcripts identified by ANOVA-PLS as differentially expressed genes (DEGs) in at least one of the experimental groups. Values were centered to the average of control samples and log2 transformed. Color scale ranges from blue (strongly underexpressed relative to control) to red (strongly overexpressed). Both rows (genes) and columns (samples) were grouped by hierarchical clustering. B, Functional enrichment analysis identified specific signaling pathways. C, qRT-PCR analysis of NKX2-5 and D, HIF-1a transcription factors during GHRH dosedependent treatment. E, qRT-PCR analysis of NKX2-5 under presence of mTOR inhibitors LY294002 and Rapamycin. F, Western blot analysis of mTOR pathway components pAkt, mTORC (Ser2448), mTORC (Ser2481) and phosphorylated tuberous sclerosis complex ( $p-$ TSC2). G, qRT-PCR analysis of NKX2-5 in HIF-1a knockout cells stimulated with GHRH 0.3uM. $\mathrm{H}$, Occupancy of NKX2-5 loci by HIF-1 $\alpha$ using genome-wide chromatin immunoprecipitation combined with high-throughput sequencing (ChIP-seq). I, Comparison of HIF-1 $\alpha$ target genes identified by ChIP-seq with changes in RNA-seq and pathway activation following HIF-1 $\alpha$ knockout in hiPSCs-CMs. J, Identification of hypoxia-response elements binding HIF-1 1 . L, Most significantly pathways affected in the absence of HIF-1a. 


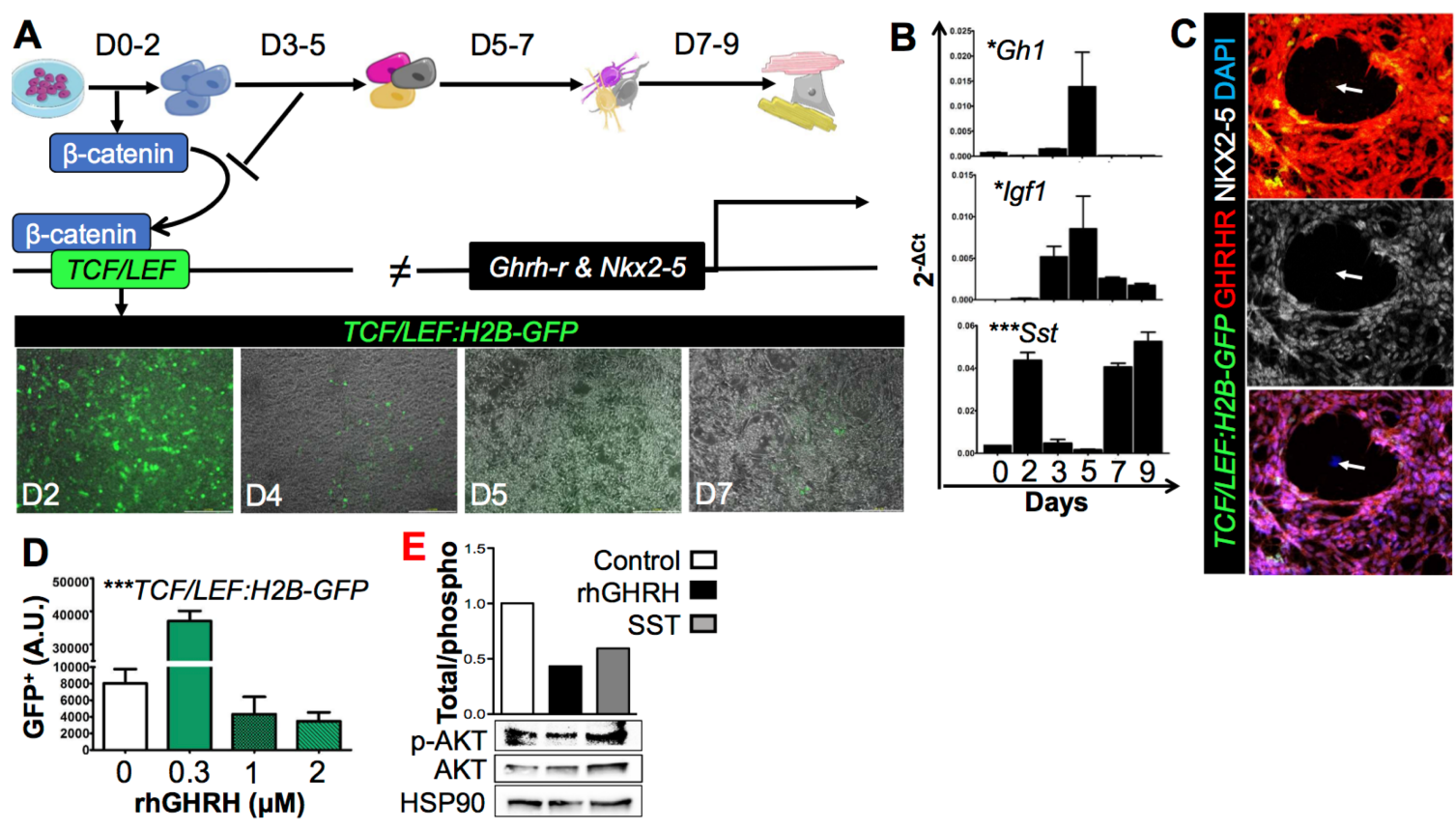

Fig.4. $\beta$-catenin stabilization and signaling of GHRH-R in atrio-ventricular cardiomyocytes. Tracking H2B-GFP reporter expressing cells in human pluripotent stem cells (hPSC) stably expressing TCF/Lef:H2B-GFP. A, Images acquired in confocal with magnification 20X on day 2, 4, 5 and 7 (D2, D4, D5 and D7). B, qRT-PCR analysis of Gh, Igf1 and SST mRNA levels in hPSC stably expressing TCF/Lef:H2B-GFP. C, Immunostaining of GHRH-R (Red) that co-localized (yellow) with H2B-GFP ${ }^{+}$cells in NKX2-5+ myocardial precursors (White). D, Quantification of H2BGFP $^{+}$cells under GHRH stimulation. E, Western blot analysis of Akt activity in hPSC expressing TCF/Lef:H2B-GFP upon GHRH or somatostatin (SST) stimulation. 


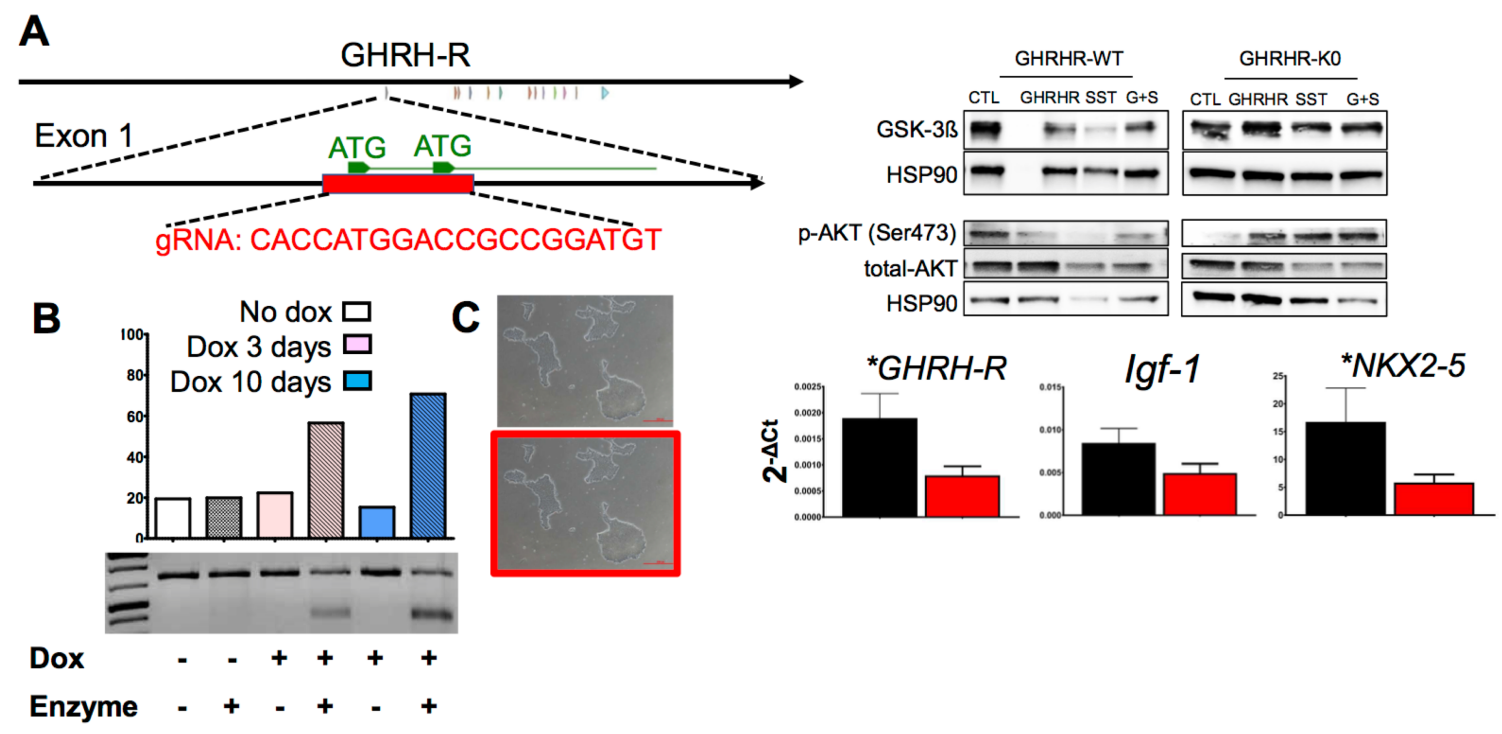

Fig.5. GHRH-R removal reduces NKX2-5 transcription. A, Schematic figure of knock-out GHRH-R signaling peptide in hPSCs expressing a tetracycline-inducible spCas9 and a gRNA spanning the ATG start sites of exon 1. Western blot analysis of Gsk-3 $\beta$ and Akt activity in hPSC wild type or knockout to GHRHR treated with GHRH, somatostatin (SST) or combination of both $(\mathrm{GHRH}+\mathrm{S})$. B, DNA mismatch cleavage detection analysis illustrating $71 \%$ efficiency in genome editing of the targeted region in response to 10-day treatment with doxycycline. C, Undifferentiated hPSCs clones after GHRHR silencing. D, qRT-PCR analysis of GHRHR, IGF1 and NKX2-5 mRNA levels in hPSC after GHRHR knockdown. 


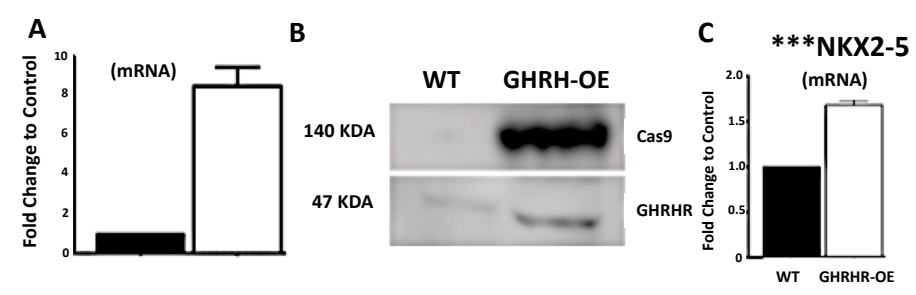

Fig.6. Sustained expression of GHRH-R enhances atrio-ventricular differentiation. A, qRT$P C R$ and $B$, Western blot analysis of GHRHR mRNA levels and protein expression in hPSC overexpressing GHRHR lentiviral vector-mediated. C, qRT-PCR analysis of NKX2-5 mRNA levels in $\mathrm{hPSC}$ overexpressing GHRHR lentiviral vector-mediated. 

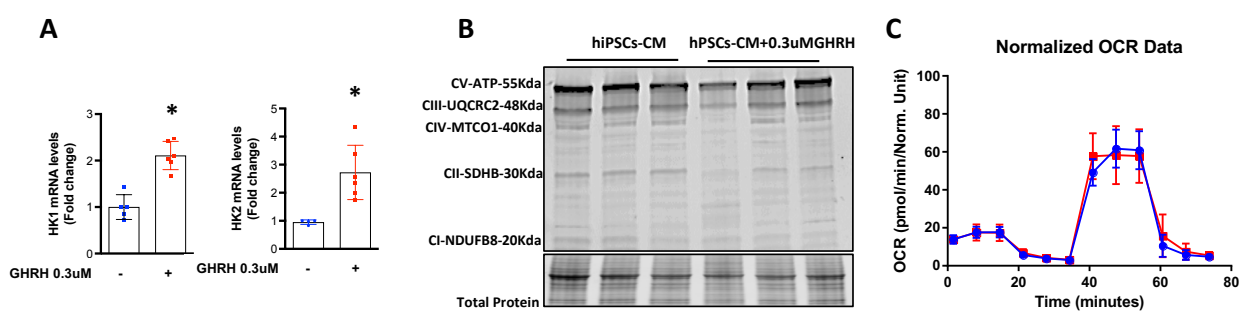

D
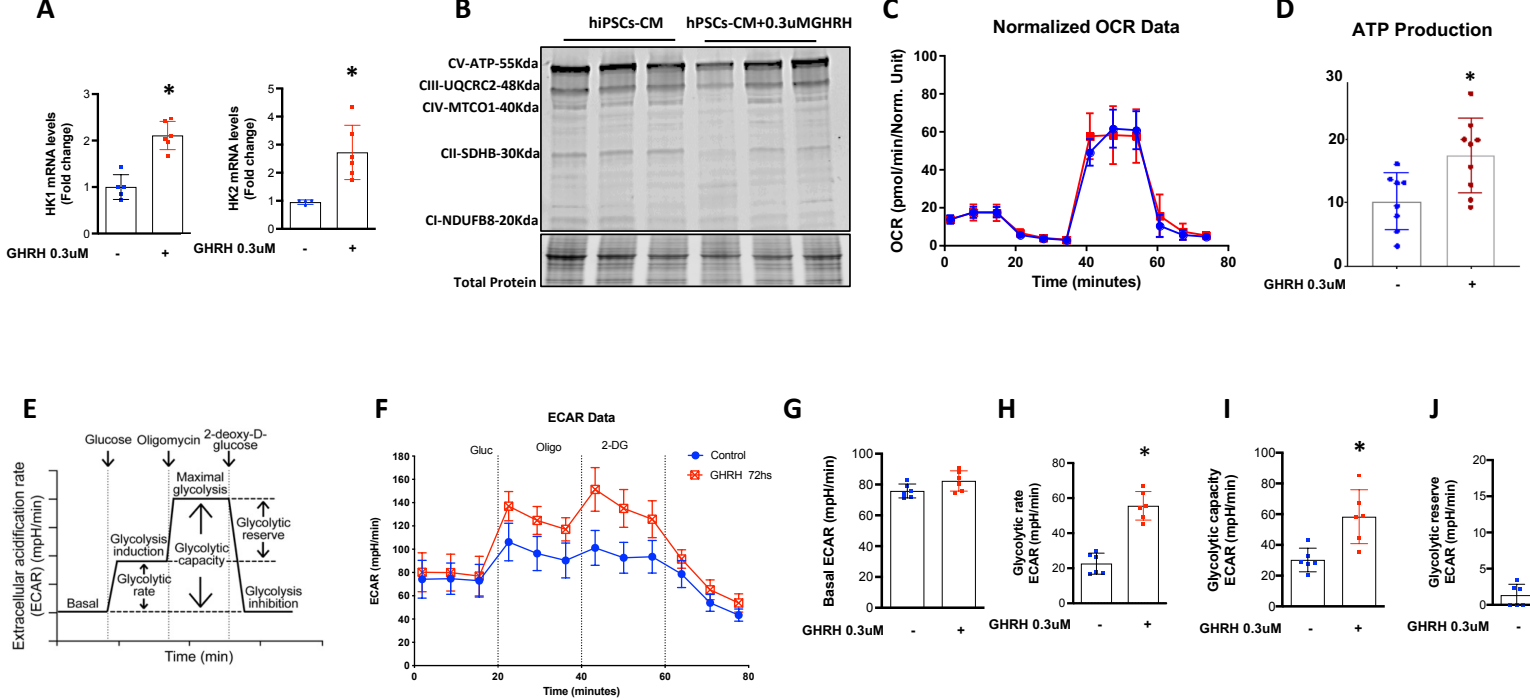

G
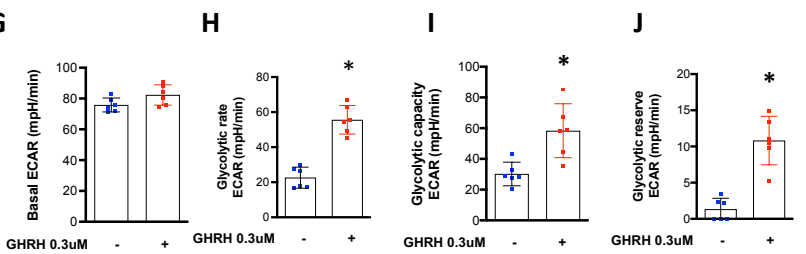

Fig.7. Activation of HIF1 $\alpha$ and Hexokinase pathway in cultured hPSC-CMs.

A, qRT-PCR analysis of hexokinase 1 (HK1) and 2 (HK2) mRNA levels in cells treated with GHRH versus non-treated; $B$, Western blot analysis of mitochondrial oxidative phosphorylation complexes; Seahorse Live-cell Metabolic Assay measurements of $\mathrm{C}$, oxygen consumption rate $(\mathrm{OCR})$; D, ATP production; F, extracelular acidification rate (ECAR) próton leak, representing glycolysis; Glycolytic rate (glycolysis induction subtracted for basal ECAR), glycolytic capacity (maximal glycolysis subtracted for basal ECAR), and glycolytic reserve (maximal glycolysis subtracted for glycolysis induction) are derived from the ECAR curve. (E) Representative kinetic study of glycolysis-dependent ECAR ( $\mathrm{mpH} / \mathrm{min})$. Bars show basal ECAR levels (left), glycolytic rate (middle left), glycolytic capacity (middle right), and glycolytic reserve (right). The data were pooled from three independent experiments; mean 6 SEM. Statistical differences were analyzed by $\mathrm{T}$ test. 
A

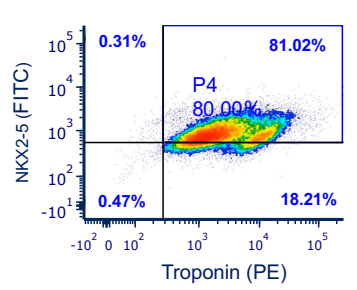

B

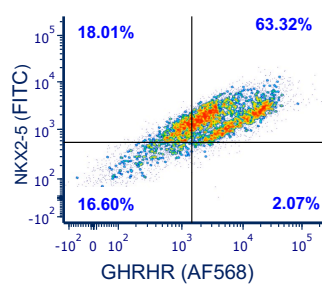

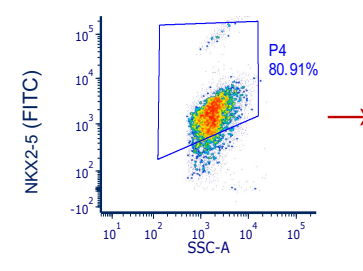

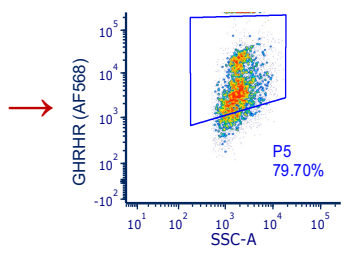

Fig.S1. Differentiation and characterization of the human pluripotent stem cells derived

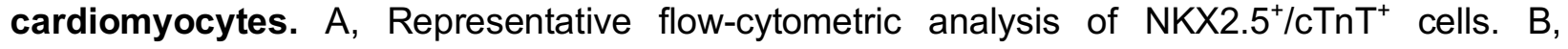
Representative flow-cytometric analyses of the proportion of $\mathrm{NKX} 2.5^{+} / \mathrm{GHRHR}^{+}$cells (left panel) and the proportion of $\mathrm{GHRHR}^{+}$cells among these $\mathrm{NKX} 2.5^{+} / \mathrm{CTnT}^{+}$cells (right panels) in day 20. Note that using cardiomyocyte differentiation protocol $\sim 18 \%$ of the differentiating cells were $\mathrm{TnT}+/ \mathrm{Nkx} 2.5^{-}$cells (sinoatrial node like cells) and that among the differentiating $\mathrm{cTnT}^{+} / \mathrm{NKX}^{2.5^{+}}$ cells obtained $\sim 80 \%$ were GHRH-R positive. 


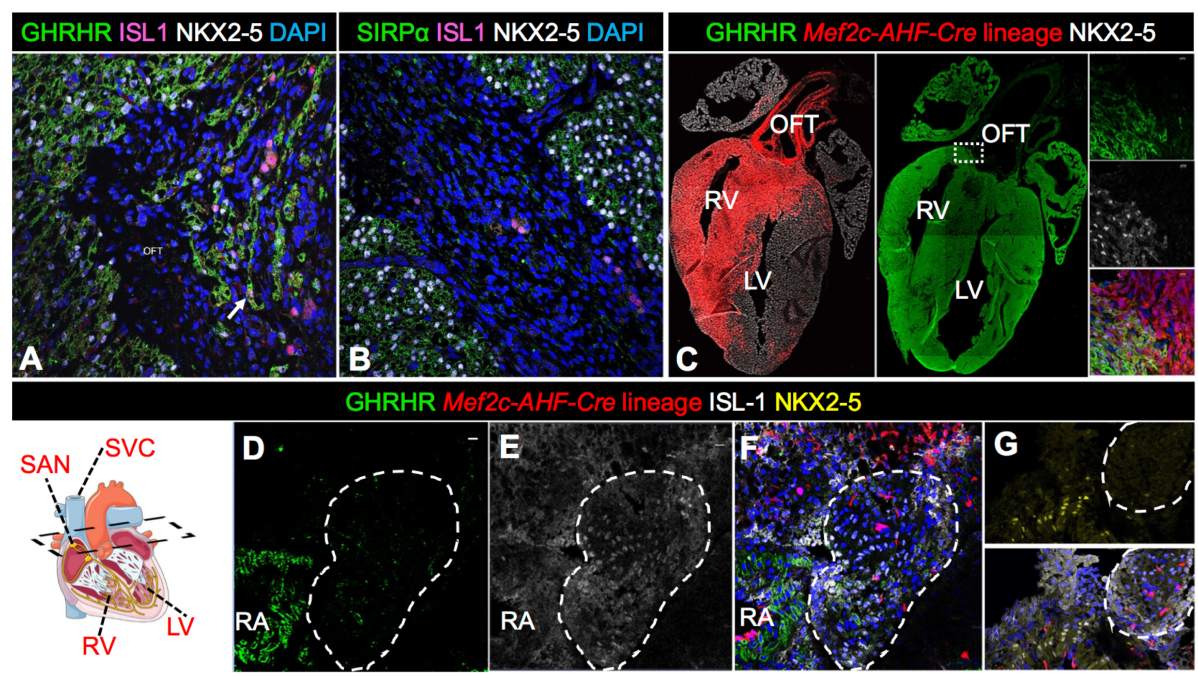

Fig.S2. GHRH-R immunoreactivity tracks with NKX2-5 in the developing human and mouse heart. A, Confocal immunofluorescence of a human fetal heart tissue at 16 weeks of gestation illustrating that GHRH-R is exclusively expressed by NKX2-5 myocardial cells, and rarely by ISL$1^{+}$cells (arrows). B, A similar pattern of immunoreactivity is observed for SIRPA (SIRPa). C, Heart-field specific analysis with a Mef2c-AHF-Cre;tdTomato mouse line, indicates that GHRH-R immunoreactivity uniformly marks atrioventricular NKX2-5 $5^{+}$cells of both FHF (tdTomato negative) and SHF (tdTomato positive) lineage. The boxed area is depicted in higher magnification in farright panels. Note that NKX2-5 cells in the outflow tract area not immunoreactive to GHRH-R. DG, A cross section of a Mef2c-AHF-Cre;tdTomato mouse heart at level of sinus node (SAN), located between the right atrium (RA) and superior vena cava (SVC). The NKX2-5+ cells in the RA exhibit GHRH-R immunoreactivity, whereas the ISL-1+ pacemaker cells do not (D-F). Note that the ISL-1 ${ }^{+}$pacemaker myocardium does not express NKX2-5. 


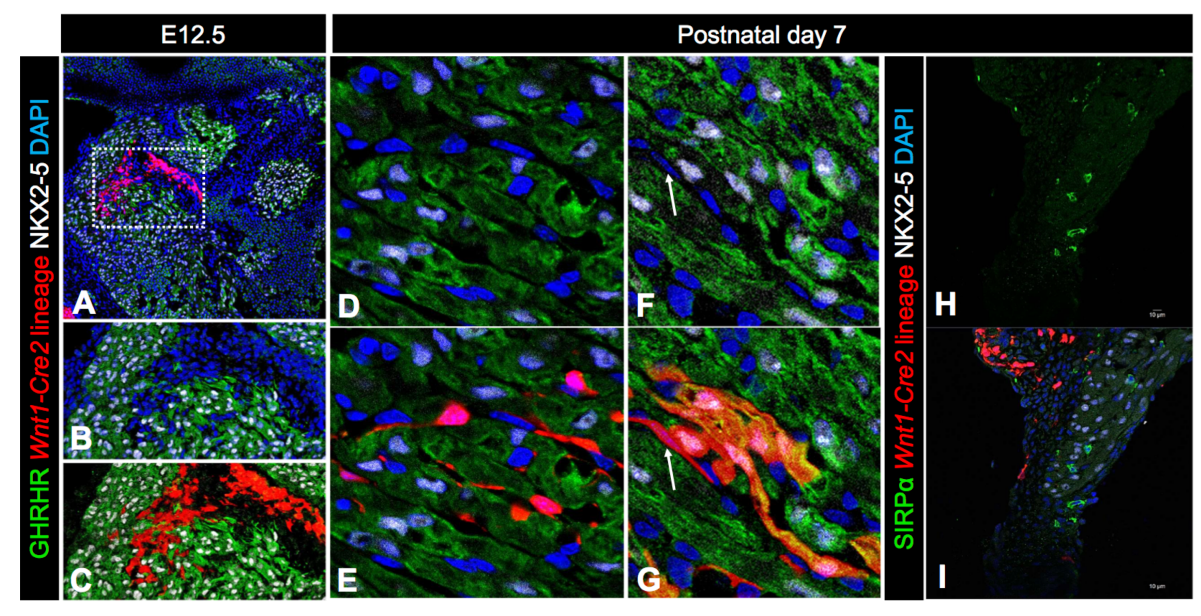

Fig.S3. A, Confocal immunofluorescence of a murine heart embryo at 12.5 weeks of gestation $5 \mathrm{x}$. The boxed area is depicted in higher magnification in the bottom panels $\mathrm{B}$ and $\mathrm{C}$. D, E, F, G, Murine heart at week 1 after birth NKX2-5+ cardiac cells (Fig. S2 $\mathrm{H}-\mathrm{I}$ ). 

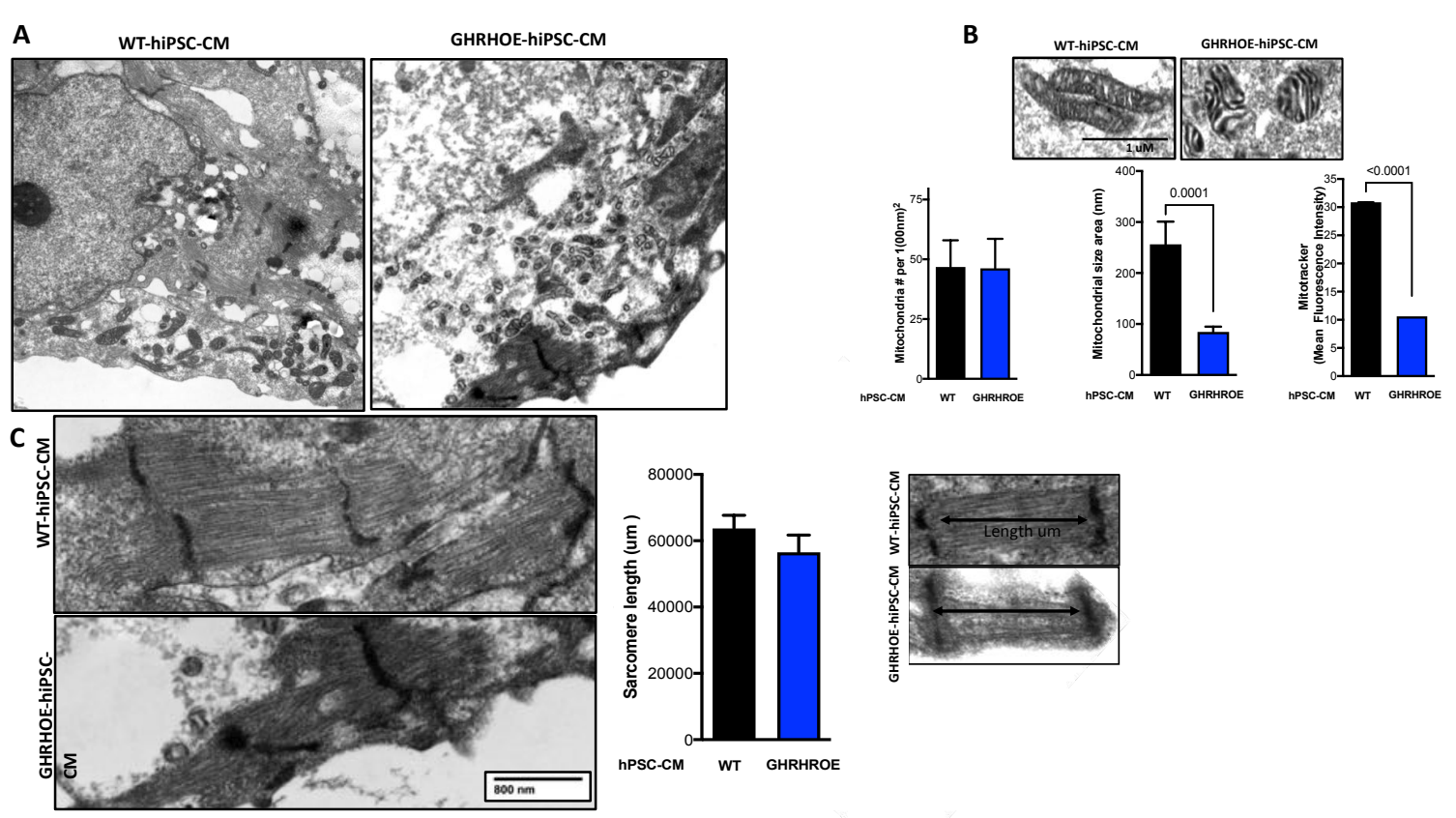

Fig.S4. hPSC-CMs overexpressing GHRHR lacks metabolic and functional maturation. A, Transmission eletron microscopy (TEM) photomicrography of a typical mitochondria in hPSC$\mathrm{CM}$; B, Quantification of mitochondrial number; C, Flow cytometric quantification of mitotracker green fluorescent staining; D, Quantification of mitochondrial size; E, Photomicrography of sarcomere and sarcomere length quantification. 


\section{Materials and Methods}

Mice

All animals were maintained in an American Association for Accreditation of Laboratory Animal Care (AAALAC)-approved animal facility at the University of Miami, Miller School of Medicine, and procedures were performed using Institutional Animal Care and Use Committee-approved protocols according to National Institutes of Health (NIH) standards. The Mef2c-AHF-Cre mice were cryorecovered at the University of Miami, Sylvester Comprehensive Cancer Center's Transgenic animal facility, from material obtained from the Mutant Mouse Regional Resource Centers (MMRRC, strain ID: 30262). The Mef2c-AHF-Cre mice were bred through the male germ line. Genotyping was performed by an independent provider via an automated real-time PCR system (Transnetyx). All analyses were performed in age-matched males and female littermates from multiple litters.

Human fetal heart tissue

Human fetal heart tissues (15 to 22 weeks of gestation) were obtained from authorized sources (Advanced Bioscience Resources Inc., Alameda, CA) following Institutional Review Board approval. Upon arrival, tissues were fixed in $10 \%$ buffered formalin for $\sim 24$ hours, embedded in paraffin, and cut into $4-$ to 5 - $\mu \mathrm{m}$-thick sections.

hPSC Differentiation toward Cardiomyocytes

For monolayer-based differentiation of cardiomyocytes (CMs), hPSCs were cultured between P30-P50 and seeded at a density of $3 \times 10^{5}$ cells/well of a 6 -well plate and grown for $48 \mathrm{hr}$ to $90 \%$ confluence in a humidified incubator with $5 \% \mathrm{CO} 2$ at $37^{\circ} \mathrm{C}$. On day 0 , the medium was replaced with RPMI 1640 supplemented with B27 without insulin and $6 \mu \mathrm{M}$ CHIR99021. On day 2, the medium was changed to RPMI 1640 supplemented with B27 without insulin for $24 \mathrm{hr}$. Day 3, medium was refreshed to RPMI 1640 supplemented with B27 without insulin and $5 \mu$ M XAV939 for another $48 \mathrm{hr}$. On day 5, the medium was changed back to RPMI-B27 without insulin for 48 $\mathrm{hr}$, and then switched to RPMI 1640 plus normal B27 until day 12 with a medium change every 2 days. From that day on, fresh RPMI 1640-B27 was changed every two days.

Immunohistochemistry and immunocytochemistry

For immunocytochemical analysis, cells were fixed in 4\% paraformaldehyde, blocked for 1 hour with $10 \%$ normal donkey serum, and processed for immunostaining as described before (10). Immunofluorescence analysis of mouse heart tissues was performed in 10- $\mu \mathrm{m}$-thick cryosections. Immunofluorescence analysis of human fetal heart samples was performed in 4- to 5- $\mu \mathrm{m}$-thick formalin fixed, paraffin-embedded tissue sections. Antigen unmasking was performed by microwaving the slides for $2 \times 10$ min in citrate buffer solution $(\mathrm{pH} \mathrm{6)}$ (Thermo Fisher Scientific). Sections were then blocked for 1 hour at room temperature with 10\% normal donkey serum (Chemicon International Inc., Temecula, CA), followed by overnight incubation at $4^{\circ} \mathrm{C}$ with the primary antibody. The following primary antibodies were used for immunohistochemistry: TBrachyury (GFP-1020); ISL-1 (Millipore Sigma-Aldrich, \#07-449); cardiac troponin-T (Alomone, \#APC052); GHRHR (Abcam, \#ab14106); anti-NKX-2.5 (Abcam, \#ab9465); SIRP-A (Novus Biologicals, \#NB300); SHOX-2 (ab10951). Subsequently, the antibodies were visualized by incubating the sections for 1 hour at $37^{\circ} \mathrm{C}$ with Alexa Fluor 488 , Alexa Fluor 568, and Alexa Fluor 633 dyes (1:500; Thermo Fisher Scientific).

\section{Western blot}

Protein lysates were prepared in radioimmunoprecipitation assay buffer and quantified with the Bradford assay (Bio-Rad). Electrophoresis was performed in precast, NuPage 4 to $12 \%$ bis-tris protein gels before transferring into polyvinylidene difluoride membranes using the TransBlot 
Turbo transfer system (Bio-Rad). Before blocking and antibody incubation, membranes were stained with Ponceau S reagent (SigmaAldrich, \#P3504) to visualize protein bands and cut at specific sizes so different antibodies could be tested at the same time. Western blots were performed using antibodies against H3K27me3 $(0.4 \mu \mathrm{g} / \mathrm{ml}$; Sigma-Aldrich, rabbit polyclonal), Isl1 (1:10,000; rabbit monoclonal, Abcam), Hsp90 (1:2000; rabbit polyclonal, Cell Signaling Technology), Gapdh (1:2000; rabbit monoclonal, Cell Signaling Technology), a goat anti-rabbit immunoglobulin G, and horseradish peroxidase- linked antibody (1:2000; Cell Signaling Technology). Densitometry analysis of Western blots was performed using Fiji ImageJ.

\section{Flow Cytometry}

The hPSC were positive for troponin, GHRHR, or NKX2-5 by flow cytometry in a BD LSR-II analyzer.

The following antibodies were used for flow cytometry: Troponin (Bioss - BS10648-PE), GHRHR (LsBio-C717859), NKX2-5 (Biorbyt - orb-103103).

\section{Quantitative PCR}

Gene expression analysis was performed as described before (10). Briefly, following total RNA extraction with the RNeasy mini kit (Qiagen) and complementary DNA synthesis with the highcapacity reverse-transcription kit (Applied Biosystems), samples were subjected to quantitative PCR in an iQ5 real-time PCR detection system (Bio-Rad), using the TaqMan Universal Master mix (Applied Biosystems). The following probes were used: Gapdh (Mm99999915), Isl1 (Mm00517585), Wnt1 (Mm01300555), and Nkx2-5 (Mm01309813); Gapdh (Hs0278624), TBP (Hs00427620), T-Brach (Hs00610080), Mesp1 (Hs00251489), Nkx2-5 (Hs00231763), Oct4 (Hs04260367), Nanog (Hs02387400), Sirpa (Hs00941118), GHRHR (Hs0181591), GHRH (Hs00184139), Isl1 (Hs00158126), Sox10 (Hs00366918), Wnt1 (Hs00180529), Pax3 (Hs07288494), Hif1a (Hs00153153), Gh (Hs07291774), Igf1 (Hs01547656), Sst (Hs00356144), Hk1 (Hs00175976), Hk2 (Hs00606086).

Overexpressing GHRHR lentiviral vector-mediated cell line sgRNA vectors targeting TGTCAGGGGACAGCAGGGGA and AGCAGAGGGTGCGGTGGAAA, of human GHRHR cDNA were individually cloned and packaged into lentiviral particles by transient transfection into H293T cells, as described previously (1). hPS cell line SBI\#1 were then transduced with sgGHRHR-containing lentiviral particles and selected with hygromycin B (50 $\mu \mathrm{g} / \mathrm{ml}) 48$ hours after initial transduction. Nonspecific sgRNA sequence was used as control.

\section{Inducible GHRHR knockdown cell line}

hPSCs with knockout of GHRHR were created by clonally isolating hPSCs stably transduced with lentiviral particles encoding spCAS9 (Addgene plasmid no. 61425) and guide RNA against GHRHR (Addgene plasmid no. 73795) directing the CRISPR-mediated deletion of the first exon of the GHRHR gene. Cells were clonally selected for optimal doxycycline-inducible GHRHR knockdown (>71\% knockdown) and expanded for use in this study. Cells were grown to $<80 \%$ confluency and induced with $1 \mu \mathrm{g} / \mathrm{mL}$ of doxycycline for 10 days. After 10 days of doxycycline induction, GHRH knockdown (GHRHKD) was confirmed by qPCR. Genomic DNA was isolated using QIAamp DNA Mini Kit (Qiagen) and RNA was isolated using TRIzol and the RNeasy Mini RNA isolation kit with RNAase-Free DNase treatment (Qiagen).

\section{RNA sequencing}

RNA was isolated with TRIzol/chloroform, DNA was further removed from samples using RNeasy MinElute Cleanup Kit (QIAGEN), according to the manufacturer's protocol. Libraries were prepared using the NEBNext Ultra kit and sequenced on the Illumina NextSeq $500(1 \times 75 \mathrm{bp})$. Next generation sequencing quality was assessed using FastQC (v0.11.3). Read counts of RNA- 
seq data were obtained using HTSeq (2) and analyzed for differential expression using EdgeR (3) with batch correction for paired experimental data. which was conducted with recommended parameters and annotated gene sets obtained from the Molecular Signatures Database (4). To ensure that our analysis was on high-quality cells, filtering was conducted by retaining cells that had unique molecular identifiers greater than 400, expressed 100 to 8000 genes.

\section{ChIP-seq and ChIP-quantitative polymerase chain reaction}

ChIP-seq and ChIP-quantitative polymerase chain reaction (qPCR) were performed on hiPSCsCMs, according to a protocol from the Cold Spring Harbor Laboratory (5), with 35 min of crosslinking. Chromatin was sonicated to an average fragment size of 200 base pairs (bp) with a Diagenode Bioruptor Pico before ChIP. A total of $5 \mu \mathrm{g}$ of antibody was used for each ChIP. The following antibodies were used to perform ChIP: HIF-1alpha (Novus), HIF-2 alpha (NB100-122). Libraries were prepared using the NEBNext Ultra kit and sequenced on the Illumina NextSeq 500 ( $1 \times 75 \mathrm{bp}$ ). ChIP-seq data were marked for duplicates using Picard (v1.128) and filtered for mapping quality using SAMtools (v1.2). Normalization of tracks was conducted using THOR, with binning set to $10 / 5$ (6). Plotting of the average ChIP-seq signals was performed using a custom script. Heat maps were generated with deeptools (7), and kmeans clustering selected to compute the matrices. Quantification of ChIP- seq data for specific regions was done using bedtools (8). Genes that did not have National Center for Biotechnology Information (NCBI) annotations were identified via a custom-built script, which performed automated alignment using BLASTN online alignment tool against the NCBI genome database. The unidentified genes were assigned a name based on the alignment score $\geq 200$.

\section{Real-time metabolic characterization}

The mitochondria oxygen consumption rate (OCR, $\mathrm{O} 2 \mathrm{mpH} / \mathrm{min})$ and extracellular acidification rate (ECAR, $\mathrm{mpH} / \mathrm{min}$ ) of $\mathrm{hPSC}$ were analyzed by an XFe96 extracellular flux analyzer (Seahorse Bioscience). For OCR studies, hPSC were harvested, washed, and resuspended in OCR medium (RPMI base, 25 mM glucose, 1 mM pyruvate, 2 mM L-glutamine [pH 7.35]). Cells (60 3 103/well) were plated in poly-D-lysine-coated 96 -well flat-bottom plates and incubated in a non-CO2 incubator for $1 \mathrm{~h}$ at $37^{\circ} \mathrm{C}$.

For ECAR analysis, hPSC were harvested, washed, and resuspended in ECAR medium (DMEM base [no bicarbonate] with $2 \mathrm{mM} \mathrm{L-glutamine,} 143 \mathrm{mM} \mathrm{NaCl}$, and $0.5 \%$ phenol red [pH 7.35]), and the complete ECAR analysis consisted of four stages: basal (without drugs), glycolysis induction (10

$\mathrm{mM}$ glucose), maximal glycolysis induction (2 mM oligomycin), and glycolysis inhibition (100 mM 2-DG).

\section{Transmission Electron Microscopy (TEM)}

CMs for TEM were fixed with glutaldehyde solution for 2 hours in room temperature and washed with cacodylate rinse buffer. Cells were then scraped from the plates and centrifuged. Following agarose processing and embedding, the samples were then sectioned, and contrast stained for imaging. TEM images were acquired at random locations throughout the samples. JEOL JEM1011 TEM was used for image acquisitions.

\section{Statistical analysis}

The scRNAseq data were analyzed in RStudio using the Seurat (40) and Monocle (26) packages, as described above. For differential expression analysis in Seurat, the default two-sided nonparametric Wilcoxon rank sum test was used. Trajectory-dependent differential expression analysis was conducted with Monocle's BEAM. Functional enrichment analysis of the DEGs was conducted with G: Profiler (https://biit.cs. ut.ee/gprofiler/gost) and GSEA (www.gsea-msigdb.org). All other statistical tests were performed in GraphPad Prism (version 8, La Jolla, CA) using 
Student's t test, Mann-Whitney test, or one-way analysis of variance (ANOVA) followed by Tukey's post hoc tests. All data met the assumptions of the tests (Bartlett's test for normality). A $P$ value of $<0.05$ was considered statistically significant. All values are reported as means \pm SEM.

1. Konermann S, Brigham MD, Trevino AE, Joung J, Abudayyeh OO, Barcena C, Hsu PD, Habib N, Gootenberg JS, Nishimasu H, Nureki O, Zhang F. Genome-scale transcriptional activation by an engineered CRISPR-Cas9 complex. Nature. 2015 Jan 29;517(7536):5838. doi: 10.1038/nature14136. Epub 2014 Dec 10. PMID: 25494202; PMCID: PMC4420636.

2. Anders S, Pyl PT, Huber W. HTSeq--a Python framework to work with high-throughput sequencing data. Bioinformatics. 2015 Jan 15;31(2):166-9. doi: 10.1093/bioinformatics/btu638. Epub 2014 Sep 25. PMID: 25260700; PMCID: PMC4287950.

3. Robinson MD, McCarthy DJ, Smyth GK. edgeR: a Bioconductor package for differential expression analysis of digital gene expression data. Bioinformatics. 2010 Jan 1;26(1):13940. doi: 10.1093/bioinformatics/btp616. Epub 2009 Nov 11. PMID: 19910308; PMCID: PMC2796818.

4. Liberzon $A$, Subramanian $A$, Pinchback $R$, Thorvaldsdóttir $H$, Tamayo $P$, Mesirov JP. Molecular signatures database (MSigDB) 3.0. Bioinformatics. 2011 Jun 15;27(12):173940. doi: 10.1093/bioinformatics/btr260. Epub 2011 May 5. PMID: 21546393; PMCID: PMC3106198.

5. Gentsch GE, Smith JC. Investigating physical chromatin associations across the Xenopus genome by chromatin immunoprecipitation. Cold Spring Harb Protoc. 2014 May 1;2014(5):pdb.prot080614. doi: 10.1101/pdb.prot080614. PMID: 24786504.

6. Allhoff M, Seré K, F Pires J, Zenke M, G Costa I. Differential peak calling of ChIP-seq signals with replicates with THOR. Nucleic Acids Res. 2016 Nov 16;44(20):e153. doi: 10.1093/nar/gkw680. Epub 2016 Aug 2. PMID: 27484474 ; PMCID: PMC5175345.

7. Ramírez F, Ryan DP, Grüning B, Bhardwaj V, Kilpert F, Richter AS, Heyne S, Dündar F, Manke T. deepTools2: a next generation web server for deep-sequencing data analysis. Nucleic Acids Res. 2016 Jul 8;44(W1):W160-5. doi: 10.1093/nar/gkw257. Epub 2016 Apr 13. PMID: 27079975; PMCID: PMC4987876.

8. Quinlan AR. BEDTools: The Swiss-Army Tool for Genome Feature Analysis. Curr Protoc Bioinformatics. 2014 Sep 8;47:11.12.1-34. doi: 10.1002/0471250953.bi1112s47. PMID: 25199790; PMCID: PMC4213956. 